hep-ph/0408320

\title{
Graviton collider effects in one and more large extra dimensions
}

\author{
Gian F. Giudice, Tilman Plehn \\ Theoretical Physics Division, CERN, CH-1211, Geneva 23, Switzerland \\ and Alessandro Strumia \\ Dipartimento di Fisica dell'Università di Pisa and INFN, Italy
}

\begin{abstract}
Astrophysical bounds severely limit the possibility of observing collider signals of gravity with less than 3 flat extra dimensions. However, small distortions of the compactified space can lift the masses of the lightest graviton excitations, evading astrophysical bounds without affecting collider signals of quantum gravity. Following this procedure we reconsider theories with one large extra dimension. A slight space warping gives a model which is safe in the infrared against astrophysical and observational bounds, and which has the ultraviolet properties of gravity with a single flat extra dimension. We extend collider studies to the case of one extra dimension, pointing out its peculiarities. Finally, for a generic number of extra dimensions, we compare different channels in LHC searches for quantum gravity, introducing an ultraviolet cutoff as an additional parameter besides the Planck mass.
\end{abstract}

\section{Introduction}

LHC experiments will hopefully allow us to understand why the electroweak scale is much smaller than the Planck scale. This hierarchy can arise from a fundamental $(4+\delta)$-dimensional Planck mass close to the electroweak scale if gravity propagates in $\delta$ extra dimensions compactified on a large volume [1]. This hypothesis has important phenomenological consequences, since high-energy colliders could probe the dynamics of gravity in its quantum and semi-classical regimes.

The prospects for observing graviton-induced processes at future collider experiments, in the case of 2 and 3 flat extra dimensions, are very much limited by the present astrophysical bounds [2]. Graviton emissions in supernovæ and neutron stars set a limit on the $D$-dimensional $(D=4+\delta)$ gravitational mass scale $M_{D}$ of about $40 \mathrm{TeV}$ for $\delta=2$ and $3 \mathrm{TeV}$ for $\delta=3$, while the limits for larger values of $\delta$ are below the $\mathrm{TeV}$ scale [2]. These bounds apparently rule out the possibility of testing the theory at the LHC for $\delta=2$, and severely restrict the available parameter space for $\delta=3$. However, it is well known that, while astrophysics probe only the infrared end of the Kaluza-Klein (KK) spectrum of gravitons, high-energy experiments are mainly sensitive to the ultraviolet side. Therefore, one can conceive non-trivial compactification spaces for which the large volume determines the hierarchy 
between the multi-dimensional and 4-dimensional Planck scales $\left(V \sim M_{\mathrm{Pl}}^{2} / M_{D}^{2+\delta}\right)$, and in which the mass of lightest KK excitation is not given by the inverse radius $1 / R \sim V^{-1 / \delta}$, but by a new intrinsic mass $\mu$. If $\mu \gtrsim 50 \mathrm{MeV}$ gravitons cannot be produced in astrophysical environments, which therefore give no bounds on the scale $M_{D}$. Whenever $\mu$ is smaller than the characteristic energy resolution of high-energy experiments, collider predictions are not affected by its presence.

Therefore, LHC quantum-gravity searches for $\delta=2$ and 3 are still viable, in spite of the strong astrophysical bounds* Undoubtedly, it is quite disturbing that the new geometrical mass scale $\mu$ has a size which is unrelated to the other scales $V^{-1 / \delta}$ or $M_{D}$, and (unless a satisfying justification is found) it appears that its only purpose is to make the theory evade the astrophysical bounds. Nevertheless, because of the great interest in investigating quantum gravity at colliders, studies of $\delta=2$ and 3 are still actively pursued.

On the other hand, the case $\delta=1$ has been discarded immediately. This is because Newtonian gravity would be modified at the macroscopic scale $M_{\mathrm{Pl}}^{2} /\left(2 \pi M_{5}^{3}\right)=\left(\mathrm{TeV} / M_{5}\right)^{3} \mathrm{AU}$, and this is excluded by astronomical observations. We have just seen that the large distance behavior of the theory can be mended by introducing an appropriate KK mass gap $\mu$ that eliminates graviton effects in astrophysics. However, this remedy does not appear immediately applicable to the case $\delta=1$. The reason is that a one-dimensional compact manifold cannot have a non-trivial shape that makes the lightest KK heavier than $1 / R$.

In this paper we show that it is indeed possible to distort the ADD model [1] with $\delta=1$ in such a way that the infrared regime coincides with Newtonian gravity up to a distance determined by the inverse of the KK mass gap $\mu$, chosen to avoid any bound from astrophysics or large-distance observations. The ultraviolet behavior of ADD with $\delta=1$ remains unmodified. Although the value of $\mu$ is chosen ad hoc for phenomenological reasons, this is completely analogous to the procedure required for the cases $\delta=2$ and 3. Therefore, LHC searches should not dismiss the case $\delta=1$ which, as we show in this paper, present interesting peculiarities.

The crucial point is that collider searches provide a test of the theory which is very robust and independent of the details of the compactification geometry. This can be simply understood by noting that the high-energy predictions are valid in the limit $V \rightarrow \infty$ (in which ordinary gravity decouples, $M_{\mathrm{Pl}} \rightarrow \infty$ ) and $\mu \rightarrow 0$ (in which the KK mass gap is neglected). High-energy collisions effectively see a $D$-dimensional infinite, non-compact and flat space. On the other hand, astrophysical bounds crucially depend on the details of the geometry and of the compactification, and are most sensitive to $\mu$ and $V$.

A second aspect of extra-dimensions discussed in this paper is the comparison between searches for graviton emission and contact interactions at the LHC. Graviton emission can be consistently computed by linearizing Einstein gravity, as long as the relevant energy is sufficiently smaller than $M_{D}$. For an $e^{+} e^{-}$collider, this condition has to be applied simply to the value of $\sqrt{s}$ at which the collider operates. But at the LHC, where parton collisions can occur at very different center-of-mass energies, some care is required [3] to define the validity region of perturbation theory.

On the other hand, unlike graviton emission, the exchange of virtual gravitons is dominated by the ultraviolet region and it is therefore not calculable in the effective Einstein theory, without specific knowledge of its short-distance completion. In ref. [4 we introduced a prescription to parametrize this ignorance in terms of an ultraviolet cutoff $\Lambda$, allowing for a possible comparison between graviton emission, tree-level graviton exchange, and graviton loops. In particular, it was shown that, for small values of $\Lambda$, direct graviton production at LEP provides the strongest constraint on $M_{D}$ while, for

\footnotetext{
${ }^{*}$ Incidentally, we should add that, on the contrary, the astrophysical bounds directly rule out the possibility of observing graviton-induced signals in experiments testing gravity in the micron-to-millimeter region.
} 
moderate and large $\Lambda$, the most stringent constraint arises from LEP limits on a dimension- 6 axialaxial effective operator which is induced by loops of gravitons. In this paper, we extend the analysis to searches at the LHC, including also the case $\delta=1$.

This paper is organized as follows. In section 2 we show how it is possible to distort one large extra dimension avoiding unwanted astrophysical effects, without affecting the signals at high-energy colliders. In section 3 we compute these signals, which are somewhat different than in the cases $\delta>1$. In section 4 we study present data, obtaining a bound $M_{D} \gtrsim 2 \mathrm{TeV}$ for $\delta=1$. In section 5 we consider LHC signals and compare the discovery reach of the different channels, for a generic number of extra dimensions $\delta$. Appendix A contains explicit formulæ for graviton effects.

\section{IR modifications of gravitons in large extra dimensions}

In this section we present a distorted version of the ADD model [1] with one extra dimension $(\delta=1)$, which has the same properties of the original model in the short-distance region (probed by colliders), but satisfies observational and astrophysical limits in the large-distance regime. The distortion introduced to forbid the unwanted low-energy effects corresponds to the RS1 model [5], in the limit in which both the compactification and the AdS radii are large with respect to the inverse of the fundamental gravity scale $M_{5}$. Pictorially, we consider a slightly warped but long extra dimension, that results in a moderately large total warp factor. The hierarchy between the Fermi and the Planck scale is generated by two factors: the large extra dimension, and warping.

We start from the RS1 model, choosing the coordinates such that the visible brane is located at $y=0$ and the Planck brane at $y=\pi R$ and the line element is

$$
d s^{2}=e^{2 \sigma(y)} \eta_{\mu \nu} d x^{\mu} d x^{\nu}+d y^{2}, \quad \sigma(y) \equiv \mu|y|
$$

with $0 \leq|y| \leq \pi R$. The 4-dimensional Planck mass is given by $\left.\right|^{\dagger}$

$$
M_{\mathrm{Pl}}^{2}=\frac{\bar{M}_{5}^{3}}{\mu}\left(e^{2 \mu R \pi}-1\right) .
$$

In the limit of small $\mu\left(\mu \ll R^{-1}\right)$, eq. (2) reduces to the usual flat-space relation $M_{\mathrm{Pl}}^{2}=\bar{M}_{5}^{3} V$, where the "volume" of the circle is $V=2 \pi R$. However, if $\mu$ is smaller than the inverse radius, it cannot really affect the KK mass gap. So we are interested in the case in which $\mu$ is larger than $R^{-1}$ (enough to damp processes in astrophysical environments), but both are much smaller than $\bar{M}_{5}$.

We expand the metric fluctuation as

$$
h_{\mu \nu}(x, y)=\sum_{n=0}^{\infty} \frac{h_{\mu \nu}^{(n)}(x)}{\sqrt{2 \pi R}} \phi^{(n)}(y),
$$

where $h_{\mu \nu}^{(n)}(x)$ has the canonical propagator as defined in ref. 3]. The KK masses are obtained by computing the eigenvalues of the operator $p_{y}^{2}$, corresponding to the square of the momentum along the fifth direction in warped space,

$$
\left[e^{-2 \sigma} \frac{d}{d y}\left(e^{4 \sigma} \frac{d}{d y}\right)+m_{n}^{2}\right] \phi^{(n)}(y)=0 .
$$

\footnotetext{
${ }^{\dagger}$ Throughout the paper $M_{\mathrm{Pl}}=2.4 \times 10^{18} \mathrm{GeV}$ is the reduced 4-dimensional Planck mass. We define $\bar{M}_{5}$ to be the reduced 5-dimensional Planck mass which, following the notation of ref. [3, is related to the Planck mass by $M_{5}=(2 \pi)^{1 / 3} \bar{M}_{5}$.
} 

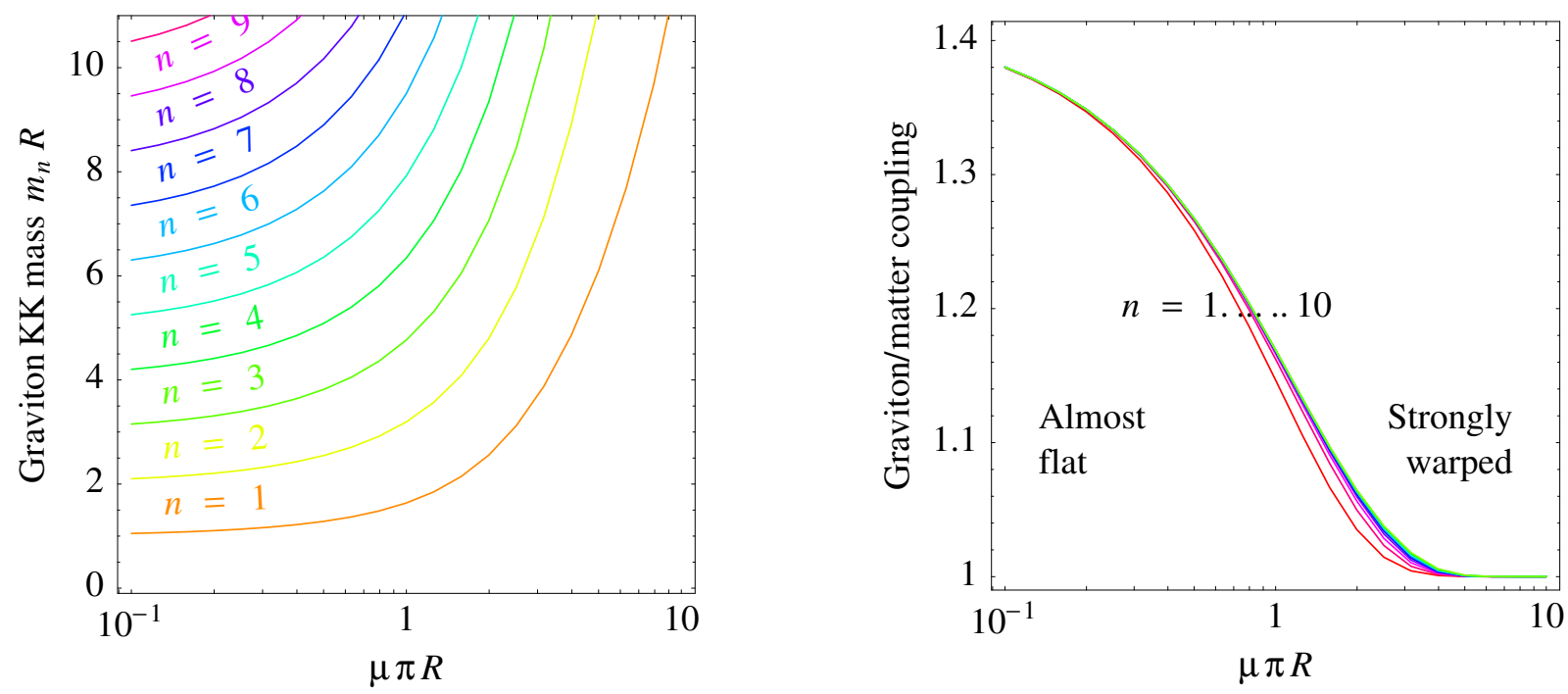

Figure 1: Masses and couplings to matter of the first 10 Kaluza-Klein excitations of gravitons, as functions of the warp factor $\mu \pi R$. The masses $m_{n}$ are given in units of $1 / R$; couplings in units of $e^{\mu \pi R} / M_{\mathrm{Pl}}$.

The graviton eigenfunctions are even, $\phi^{(n)}(-y)=\phi^{(n)}(y)$, satisfy the boundary conditions $d \phi^{(n)} / d y=$ 0 at $y=0$ and at $y=\pi R$, and the orthonormality relation $\int_{-\pi R}^{\pi R} d y e^{2 \sigma} \phi^{(n)}(y) \phi^{(m)}(y)=2 \pi R \delta_{n m}$.

The solutions to eq. (4) are

$$
\phi^{(n)}(y)=\frac{z_{n}^{2}}{N_{n}}\left[J_{2}\left(z_{n}\right)+c_{n} Y_{2}\left(z_{n}\right)\right], \quad z_{n} \equiv \frac{m_{n}}{\mu} e^{-\sigma(y)},
$$

where $J_{\nu}$ and $Y_{\nu}$ are Bessel function ${ }^{\ddagger}$. The integration constants $N_{n}$ and $c_{n}$ are determined by the orthonormality condition and by the first boundary condition

$$
\begin{gathered}
N_{n}^{2}=\frac{2 a_{n}^{2}}{\mu R \pi^{3}}\left[\frac{1}{Y_{1}^{2}\left(a_{n}\right)}-\frac{1}{Y_{1}^{2}\left(b_{n}\right)}\right], \quad c_{n}=-\frac{J_{1}\left(a_{n}\right)}{Y_{1}\left(a_{n}\right)}, \\
a_{n} \equiv \frac{m_{n}}{\mu}, \quad b_{n} \equiv a_{n} e^{-\mu R \pi} .
\end{gathered}
$$

The second boundary condition determines the masses $m_{n}$ from the equation

$$
J_{1}\left(a_{n}\right) Y_{1}\left(b_{n}\right)=J_{1}\left(b_{n}\right) Y_{1}\left(a_{n}\right) .
$$

The interactions of massive gravitons are described by the Lagrangian density

$$
\mathscr{L}=-\frac{1}{\bar{M}_{5}^{3 / 2}} \int d y h_{\mu \nu}(x, y) T^{\mu \nu}(x) \delta(y)=-T^{\mu \nu} \sum_{n=1}^{\infty} \frac{1}{\Lambda_{n}} h_{\mu \nu}^{(n)}(x),
$$

\footnotetext{
${ }^{\ddagger}$ We list some properties of the Bessel functions we used to obtain our results:

$$
\begin{gathered}
J_{\nu+1}(x) Y_{\nu}(x)-J_{\nu}(x) Y_{\nu+1}(x)=2 /(\pi x), \quad J_{\nu+2}(x) Y_{\nu}(x)-J_{\nu}(x) Y_{\nu+2}(x)=4(\nu+1) /\left(\pi x^{2}\right), \\
J_{1}(x) \stackrel{x \rightarrow \infty}{\simeq} \sqrt{2 /(\pi x)} \cos (x-3 \pi / 4), \quad Y_{1}(x) \stackrel{x \rightarrow \infty}{\simeq} \sqrt{2 /(\pi x)} \sin (x-3 \pi / 4), \\
J_{1}(x) \stackrel{x \rightarrow 0}{\simeq} x / 2, \quad Y_{1}(x) \stackrel{x \rightarrow 0}{\simeq}-2 /(\pi x) .
\end{gathered}
$$
}


with

$$
\Lambda_{n} \equiv \frac{\bar{M}_{5}^{3 / 2} \sqrt{2 \pi R}}{\phi^{(n)}(0)}
$$

Using eqs. (2) and (5), the graviton interaction scale can be written as

$$
\Lambda_{n}=M_{\mathrm{Pl}} \sqrt{\frac{1-J_{1}^{2}\left(a_{n}\right) / J_{1}^{2}\left(b_{n}\right)}{e^{2 \mu R \pi}-1}},
$$

and it is shown in fig. 1. where we plot the masses and the matter couplings of the first $10 \mathrm{KK}$ excitations of the graviton as functions of the warp factor. We see that warping removes the lightest KK's with mass $\sim 1 / R$ from the spectrum.

The expressions written so far are valid for any value of $\mu$. First consider the limit of small AdS inverse radius $\mu \ll R^{-1}$, in which eq. (8), determining the mass eigenvalues, becomes $\sin \left(m_{n} R \pi\right)=0$. This leads to the equally-spaced KK masses $m_{n}=n / R$, characteristic of flat space. In the same limit, eq. (11) becomes $\Lambda_{n}=M_{\mathrm{Pl}} / \sqrt{2}$, recovering the ADD result $\$$.

However, we are interested here in the case $\mu>R^{-1}$. Then eq. (8) is approximately equal to $J_{1}\left(a_{n}\right)=0$, whose solutions are $m_{n}=x_{n} \mu$ with

$$
x_{1} \approx 3.8, \quad x_{2} \approx 7.0, \quad x_{n}=\beta_{n}-\frac{3}{8 \beta_{n}}+\frac{3}{128 \beta_{n}^{3}}-\frac{1179}{5120 \beta_{n}^{5}}+\ldots, \quad \beta_{n} \equiv\left(n+\frac{1}{4}\right) \pi .
$$

Notice that at large $n$ KK states are again equally spaced.

For a given KK mass gap $m_{\mathrm{GAP}}=m_{1}$, we obtain

$$
\begin{gathered}
\mu=\left(\frac{m_{\mathrm{GAP}}}{50 \mathrm{MeV}}\right) 13 \mathrm{MeV} \\
R=\frac{1}{2 \pi \mu} \ln \frac{\mu M_{\mathrm{Pl}}^{2}}{\bar{M}_{5}^{3}}=\left(\frac{50 \mathrm{MeV}}{m_{\mathrm{GAP}}}\right)\left[1.9+1.2 \times 10^{-2} \ln \left(\frac{m_{\mathrm{GAP}}}{50 \mathrm{MeV}}\right)\left(\frac{\mathrm{TeV}}{\bar{M}_{5}}\right)^{3}\right] \mathrm{MeV}^{-1} .
\end{gathered}
$$

Therefore, $\mu$ is the parameter which controls the mass gap and its minimum value is determined by the astrophysical bounds. The KK mass gap is large enough to avoid clash with observations, but still small enough to be unessential in the ultraviolet region where high-energy experiments are performed. The warp factor $\exp (\mu R \pi) \sim M_{\mathrm{Pl}} \mu^{1 / 2} / \bar{M}_{5}^{3 / 2}$ is large, but much smaller than in RS1, where $\exp (\mu R \pi) \sim M_{\mathrm{Pl}} / \bar{M}_{5}$.

For $\mu>R^{-1}$, the graviton interaction scale becomes $\Lambda_{n}=M_{\mathrm{Pl}} \exp (-\mu R \pi)$, which can be expressed as

$$
\Lambda_{n} \simeq \frac{\bar{M}_{5}^{3 / 2}}{\mu^{1 / 2}}=\left(\frac{\bar{M}_{5}}{\mathrm{TeV}}\right)^{3 / 2}\left(\frac{50 \mathrm{MeV}}{m_{\mathrm{GAP}}}\right)^{1 / 2} 2.8 \times 10^{5} \mathrm{GeV}
$$

Notice that both in the limit of small or large $\mu R$, the interaction scale $\Lambda_{n}$ is independent of $n$ and all KK gravitons have a universal coupling. This is not true in the intermediate region, as illustrated in fig. 1.

If the energy resolution in a collider experiment is larger than the graviton KK mass splitting $(\sim \pi \mu)$, it is more appropriate to consider inclusive processes, in which we sum over the individual contributions of the KK modes. Using a continuous, instead of a discrete, description of the KK spectrum, the density of states in the original ADD model with $\delta=1$, expressed in terms of the KK masses $m$, is

$$
d n=R d m=\frac{M_{\mathrm{Pl}}^{2}}{2 \pi \bar{M}_{5}^{3}} d m .
$$

\footnotetext{
${ }^{\S}$ We are summing only over positive KK indices $n$, and this explains the extra $\sqrt{2}$ factor.
} 
In the case of the distorted ADD model under consideration, we find

$$
d n=\left(\frac{d x_{n}}{d n}\right)^{-1} \frac{d m}{\mu}, \quad \frac{d x_{n}}{d n}=\pi\left(1+\frac{3}{8 \beta_{n}^{2}}+\ldots\right)
$$

We write the $n$-th KK graviton production cross section as $\hat{\sigma}_{n} / \Lambda_{n}^{2}$, where we have factored out the interaction mass scale defined in eq. (9). We obtain that the inclusive cross section, both in the case of undistorted and distorted ADD is given by

$$
\sum_{n=1}^{\infty} \frac{\hat{\sigma}_{n}}{\Lambda_{n}^{2}}=\frac{1}{\pi \bar{M}_{5}^{3}} \int_{0}^{\infty} d m \hat{\sigma}(m) .
$$

Therefore, although in the distorted model the state distribution is less dense, the KK modes interact more strongly than in the undistorted model, in such a way that the two effects exactly compensate in inclusive cross sections. The result is easily understood: if a collider experiment is not sensitive to the aspects of the discretization (i.e. if the energy resolution is larger than both $\mu$ and $1 / R$ ), the two models are completely indistinguishable. The two models differ only in the far infrared, where astrophysical probes are important.

The equivalence between the two models holds also for effective interactions mediated by gravitons, as long the typical energy of the relevant physical process is larger than the KK mass gap (and we are allowed to go from the discrete to the continuum). For instance, graviton tree-level exchange in the $s$-channel leads to the scattering amplitude

$$
\begin{gathered}
\mathcal{A}=\mathcal{S}(s) \mathcal{T}, \quad \mathcal{T} \equiv T_{\mu \nu} T^{\mu \nu}-\frac{1}{3} T_{\mu}^{\mu} T_{\nu}^{\nu} \\
\mathcal{S}(s)=\sum_{n=1}^{\infty} \frac{1}{\Lambda_{n}^{2}} \frac{1}{s-m_{n}^{2}+i \varepsilon}=\frac{1}{\pi \bar{M}_{5}^{3}} \int_{0}^{\infty} \frac{d m}{s-m^{2}+i \varepsilon} .
\end{gathered}
$$

which agrees with the usual definition in $D$ dimensions [3]

$$
\mathcal{S}(s)=\frac{1}{M_{D}^{2+\delta}} \int_{-\infty}^{+\infty} \frac{d^{\delta} q}{s-q^{2}+i \varepsilon}
$$

\section{Extensions to $\delta>1$}

The deformation of the $\delta=1$ ADD model presented above can be generalized to $\delta>1$. Particularly interesting is the case $\delta=2$, where a distortion is needed to confront the strong astrophysical bounds.

The simplest generalization can be obtained by following the construction described in ref. [6]. Let us consider $\delta$ (with $\delta>1$ ) non-parallel $(2+\delta)$ branes which live in a $(4+\delta)$ dimensional space. Each brane has co-dimension 1 , and therefore for each one we can construct an RS model with an AdS mass $\mu$ which acts as a mass gap. The intersection of the branes, which has dimension $(2+\delta)-(\delta-1)=3$, is taken to be the ordinary space where the SM fields are confined. In this case, one can explicitly compute the mass spectrum in presence of the distortion parameter $\mu$. Another possibility is to start from the 6-dimensional models with gravity localized on string-like defects [7].

For $\delta>1$, one can use an alternative approach and consider non-trivial compactification spaces, as the compact hyperbolic manifold, considered in ref. [8]. In this case, the volume-radius ratio $V / R^{\delta}$ is a coefficient determined by the topology, which can be much larger than one. By making an appropriate choice of the largest distance inside the manifold, it is possible to arbitrarily increase the KK mass gap, for a fixed value of the volume $V$. 
A different example [9] is given by a general 2-dimensional torus determined by its volume $V$, by the ratio between the two radii $r$ (choosing $r \leq 1$ ), and by the shift angle $\theta$ (with $0<\theta \leq \pi / 2$ ). The KK mass spectrum is given by

$$
m_{n}^{2}=\frac{4 \pi^{2}}{V \sin \theta}\left(n_{1}^{2} r+\frac{n_{2}^{2}}{r}-2 n_{1} n_{2} \cos \theta\right)
$$

where $n_{1,2} \in \mathbf{Z}$. For $\theta=\pi / 2$, the mass square of the first $\mathrm{KK}$ mode is $4 \pi^{2} r / V$, and therefore departing from the standard value $r=1$ can only reduce the mass gap. When $\theta \rightarrow 0$, it is always possible to find values of $n_{1,2}$ such that the term inside brackets in eq. (22) vanishes, as long as $r$ is rational. Therefore, for rational $r$, the mass gap goes to zero as $\theta \rightarrow 0$. According to ref. [9], an interesting twist arises when $r$ is an algebraic irrational number. In this case, for $\theta \rightarrow 0$, the mass gap determined by eq. (22) goes to a constant, which can then be chosen arbitrarily large, for any fixed value of $V$. For a careful choice of parameters, one can appropriately tune the mass gap to the preferred value of $\mu$.

\section{Graviton signals in one large extra dimension}

The distortion introduced in the previous section to cure the low-energy phenomenology of gravitons in large extra dimensions does not affect their high-energy signals. Therefore we can study them by setting $\delta=1$ in the expressions, valid for a generic number of extra dimensions, for graviton-emission processes [3] and graviton-loop induced interactions [4]. An interesting peculiarity of $\delta=1$ occurs in processes generated by tree-level graviton exchange. Contrary to the cases with more extra dimensions, the integral in eq. 21) is not divergent in the ultraviolet. For $\delta=1$ the integral is dominated by KK masses of the order of $\sqrt{s}$. Introducing an ultraviolet cutoff $\Lambda$, which corresponds to the largest KK graviton mass, we obtain

$$
\mathcal{S}(s)=\frac{2}{M_{5}^{3} \sqrt{s}} \operatorname{arctanh} \frac{\Lambda}{\sqrt{s}} \simeq \frac{1}{M_{5}^{3}}\left(\frac{-i \pi}{\sqrt{s}}+\frac{2}{\Lambda}\right) .
$$

In the limit $\Lambda \rightarrow \infty, \mathcal{S}(s)$ is actually purely imaginary, and therefore there is no interference with Standard Model contributions to the same physical process. The imaginary part in $\mathcal{S}(s)$ corresponds to the exchange of a resonant graviton, as can be seen by giving a small width $\Gamma$ to the KK excitations. The physical value of the width can be smaller or larger than the separation between consecutive KK, giving rise to a series of separated or of overlapped resonances. Studying experiments that do not have enough energy resolution to see the difference, it is convenient to take the simpler limit $\Gamma \rightarrow 0$, where

$$
\lim _{\Gamma \rightarrow 0} \frac{1}{s-m^{2}+i m \Gamma}=P\left(\frac{1}{s-m^{2}}\right)-i \pi \delta\left(s-m^{2}\right) .
$$

Physically this means that at any $s$ the dominant effect is on-shell production of the KK mode with mass $m=\sqrt{s}$. This is why graviton exchange does not interfere with the SM amplitude, as mathematically accounted by the $i$ factor in eq. 23). The unknown physics that acts as cut-off of quantum gravity can give the uncontrollable extra contribution suppressed by $\Lambda$ in eq. (23), which can be real and change the above conclusion.

Effects mediated by virtual graviton exchange in the $t$-channel are qualitatively different, because now gravitons cannot be resonantly produced. Indeed, inserting $t<0$ in the expression for $\mathcal{S}$, eq. (23), we get that the $t$-channel contribution is purely real, $\mathcal{S}(t)<0$. Therefore the $t$-channel contribution can interfere with the SM amplitude. When the SM cross section is dominant, $t$-channel graviton exchange gives the only significant correction. Consequently particular attention should be paid to 


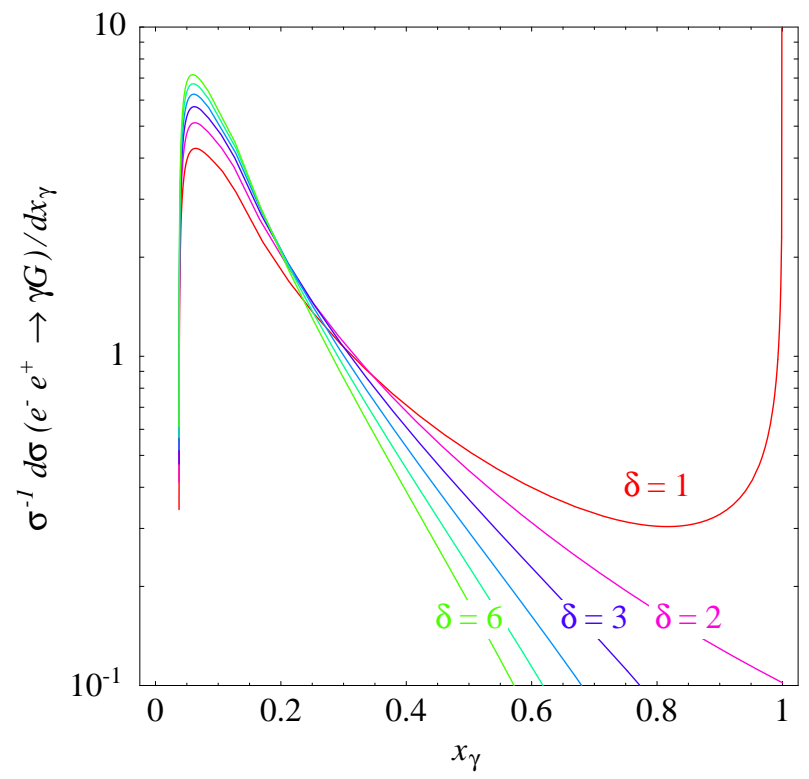

Figure 2: Photon spectrum $\left(x_{\gamma}=2 E_{\gamma} / \sqrt{s}\right)$ in $e^{+} e^{-} \rightarrow \gamma G$ collisions for different numbers of extra dimensions, $\delta=1,2,3,4,5,6$. We included the typical cuts performed by LEP collaborations ( $p_{T}^{\gamma}>$ $0.0365 \sqrt{s}$ and $\left.\left|\cos \theta_{\gamma}\right|<0.95\right)$.

elastic processes, like Bhabha scattering at LEP, electron-jet final states at HERA, and dijet production at Tevatron and LHC.

The dependence of $\mathcal{S}$ on the kinematical variables of the process signals a non-local effect. This can be interpreted as the result of a large anomalous dimension of the local dimension- 8 operator $\mathcal{T}$ corresponding to the linear (instead of logarithmic) running in 5 dimensions. Effectively, this turns $\mathcal{T}$ into a dimension- 7 operator, suppressed only by 3 powers of $\bar{M}_{5}$, enhancing the effect of tree-level graviton exchange. Despite the IR enhancement in eq. (23), the graviton contribution still grows with the collider energy. However, at fixed energy, forward elastic scattering events (i.e. small $|t|$ ) are statistically as significant as hard scattering events (i.e. $t \sim-s$ ). This can be contrasted to the $\delta>1$ case, where the SM background hides graviton effects in forward elastic scattering.

The case $\delta=1$ is rather special, because of the potential sensitivity to the infrared. New infrareddominated phenomena can occur if, besides gravitons, other massless (non-derivatively coupled) scalar or vector particles exist in the bulk. For instance, the tree-level exchange of a massless bulk scalar particle coupled to a constant source on the Planck brane can give corrections to parameters on the visible brane proportional to $\bar{M}_{5} \int d k_{5} / k_{5}^{2} \sim \bar{M}_{5} / \mu$, where $\mu$ is the infrared cutoff. It was argued in ref. [10] that this effect can destabilize the hierarchy in the limit of a large compactification radius $R$, whenever $\mu=1 / R$.

Suppose that a new $U(1)$ gauge field exist in the bulk. This can mix in the kinetic term with the hypercharge gauge field, $\epsilon F_{\mu \nu}^{Y} F^{(X) \mu \nu}$, where $\epsilon$ is some effective coupling constant. The tree-level exchange of the $X$ gauge KK modes induces a running of $\alpha$ linear (and not logarithmic) with energy proportional to $\epsilon$. Here we assume the absence of such scalar or vector bulk massless particles. 

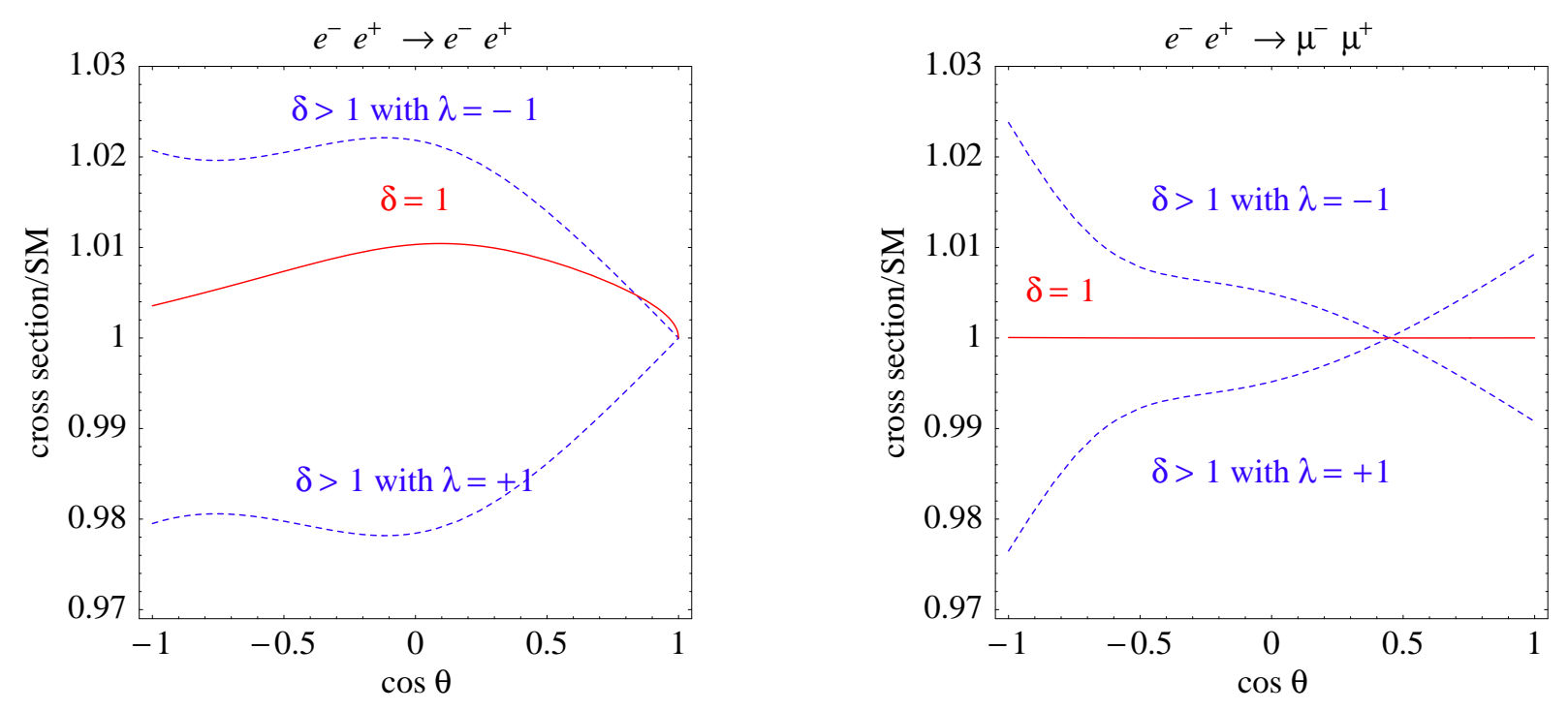

Figure 3: Corrections to $e^{+} e^{-} \rightarrow e^{+} e^{-}$(left) and $e^{+} e^{-} \rightarrow \mu^{+} \mu^{-}$(right) cross sections due to tree-level virtual exchange of gravitons with $\delta=1$ (red continuous line, for $M_{D}=2.5 \mathrm{TeV}$ ) and $\delta>1$ (blue dashed lines, for $\mathcal{S}=8 \lambda /(1.25 \mathrm{TeV})^{4}$ and $\left.\lambda= \pm 1\right)$. In the latter case the result depends on the $U V$ cut-off, so that not even its sign can be reliably computed: we consider the two cases of constructive and destructive interference with the SM. For $\delta=1$ the effect is unambiguously fixed: it increases the Bhabha cross section, and gives a negligible correction to inelastic scatterings.

\section{Comparison with present data}

Presently, LEP experiments are the most sensitive probes of gravitons in one large extra dimension. We consider three kinds of graviton effects: 1) graviton emission; 2) tree-level virtual exchange of gravitons; 3) one-loop virtual exchange of gravitons.

1) Graviton emission. LEP experiments measured the energy and angular spectrum of $e^{+} e^{-} \rightarrow \gamma$ + missing energy events at $\sqrt{s} \approx 200 \mathrm{GeV}$. The predicted $d^{2} \sigma / d E_{\gamma} d \cos \theta_{\gamma}$ (where $E_{\gamma}$ and $\theta_{\gamma}$ are the energy and direction of the photon in the center-of-mass frame) was computed in ref. [3] and is here reported in appendix $\mathrm{A}$ and plotted in fig. 2 for different values of $\delta$. For $\delta>1$ the bounds are dominated by data at small values of $E_{\gamma} \ll \sqrt{s}$ (where there is some SM background) because the signal is strongly suppressed at the highest photon energies $\left(E_{\gamma}\right.$ close to $\left.\sqrt{s} / 2\right)$, where the SM background is negligible. Indeed the differential cross section is divergent when $E_{\gamma} \rightarrow 0$ or $\left|\cos \theta_{\gamma}\right| \rightarrow 1$, because of the familiar massless electron exchange in the $t$ channel. The graviton signal decreases with $E_{\gamma}$ more slowly as the value of $\delta$ is increased.

A novelty specific to the case $\delta=1$ is the rise around the largest values of $E_{\gamma}$. Actually, the differential cross section is divergent for $E_{\gamma} \rightarrow \sqrt{s} / 2$ (see fig. 2). Such divergence corresponds to emission of KK gravitons with very small mass and is present because we approximated the dense KK graviton spectrum with a continuum. This approximation is valid because the integrated cross section remains finite.

By imposing $\sigma\left(e^{+} e^{-} \rightarrow \gamma G\right)<0.01 \mathrm{pb}^{-1}$ at $\sqrt{s}=207 \mathrm{GeV}$ with the following cuts

$$
E_{\gamma}>0.7 \sqrt{s}, \quad p_{T}^{\gamma}>0.0365 \sqrt{s}, \quad\left|\cos \theta_{\gamma}\right|<0.95,
$$




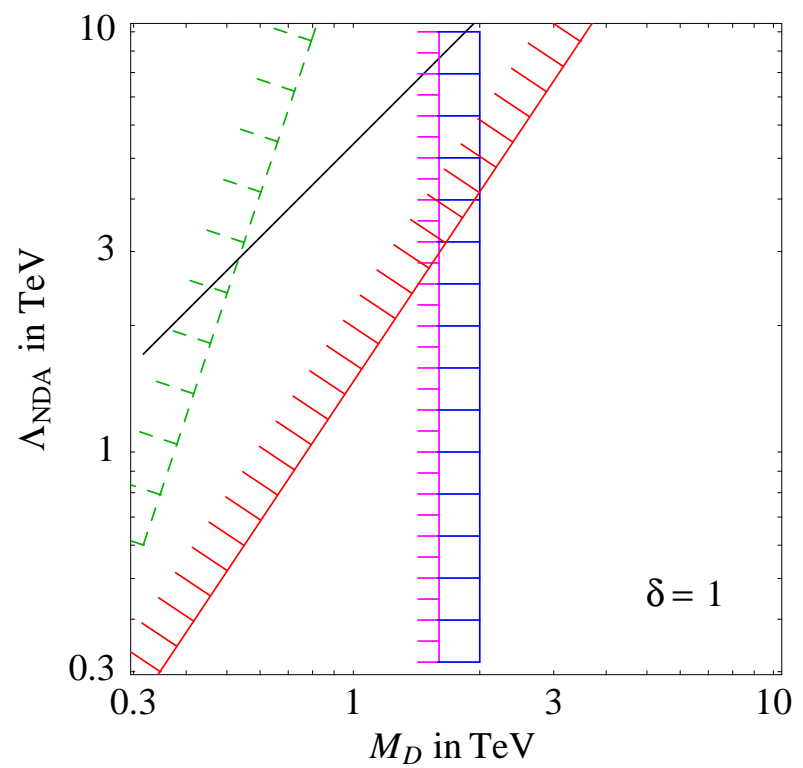

Figure 4: $95 \% C L$ collider bounds on graviton phenomenology in the plane $\left(M_{D}, \Lambda_{\mathrm{NDA}}\right)$ for $\delta=1$ flat large extra dimension. The solid black line shows the value of the cut-off $\Lambda_{\mathrm{NDA}}$ corresponding to a strongly-interacting gravitational theory. The other lines mark the regions excluded by the bounds from graviton emission (vertical blue line), tree-level virtual graviton exchange (purple vertical line with short borderlines), graviton loops (red solid line), graviton and gauge boson loops (green dashed line).

we obtain the bound $M_{D}>2.4 \mathrm{TeV}$. This requirement roughly corresponds to less than 10 events in all LEP2 experiments. A precise analysis can only be performed by the experimental collaborations.

For $\delta>1$ the process $e^{+} e^{-} \rightarrow \gamma G$ gives a more significant probe of graviton emission than $e^{+} e^{-} \rightarrow Z G$. This is because the signal is maximal in the kinematical region where the graviton is heaviest, and the $Z$ mass limits the accessible region of graviton masses. As previously discussed, the case $\delta=1$ is special, since a significant contribution to the signal comes from the region of light gravitons. Therefore, for $\delta=1$ one can take full advantage of the coupling of the $Z$ to electron, which is stronger than the $\gamma$ coupling. Appendix A contains our result for the cross section, in agreement with previous studies [11. The L3 limit on the total cross section, $\sigma(e \bar{e} \rightarrow Z G)<0.29 \mathrm{pb}$ at $95 \%$ C.L. [12], implies $M_{D}>1.4 \mathrm{TeV}$ for $\delta=1$. This limit is subdominant, but using up-to-date LEPcombined data on the differential $e \bar{e} \rightarrow Z G$ cross section (presently not available) might give a probe competitive with other processes.

2) Tree-level virtual graviton exchange. As explained in section 3 and illustrated in fig. 3 . virtual effects mediated by tree level exchange of gravitons in $\delta=1$ extra dimension are qualitatively different than in the other cases. For $\delta>1$ these effects are UV divergent, so that it is only possible to estimate them by introducing an arbitrary cut-off $\Lambda$, which becomes more and more important for bigger $\delta$. Only in the case $\delta=1$ these effects can be reliably computed, and used to set a constraint on $M_{D}$. In appendix $\mathrm{A}$ we give our results for the cross sections of the most interesting processes at electron-positron and hadron colliders. When specialized to $\delta>1$ some of our results do not fully agree with previous literature. For $\delta=1$ Bhabha scattering is the only relevant LEP probe [13]. We find that it sets the $99 \%$ C.L. bound $M_{D} \gtrsim 2.4 \mathrm{TeV}$. This value is somewhat higher than the sensitivity of the experiments, $M_{D}=2.0 \mathrm{TeV}$ at $99 \%$ C.L., because the data show a moderate preference (almost 
$2 \sigma$ ) for an effect with the opposite sign. A precise analysis can only be done by the experimental collaborations. (Notice that setting $\delta=1$ in codes developed for studying $\delta>1$ is not correct.)

3) One-loop virtual graviton exchange. The effects of graviton loops can become more important than tree-level exchange because they generate effective operators with dimensions lower than $\mathcal{T}$. As discussed in ref. [4, especially important is the operator

$$
\mathscr{L}=c_{\Upsilon} \Upsilon, \quad \Upsilon=\frac{1}{2}\left(\sum_{f} \bar{f} \gamma_{\mu} \gamma_{5} f\right)\left(\sum_{f} \bar{f} \gamma^{\mu} \gamma_{5} f\right),
$$

where the sum is over quarks and leptons, and the coefficient $c_{\Upsilon}$ is flavour-universal. Using the rules of naïve-dimensional analysis presented in ref. [4], we estimate

$$
c_{\Upsilon}=\frac{1}{16 \pi^{2}} \frac{\Lambda_{\mathrm{NDA}}^{4}}{M_{5}^{6}}
$$

where $\Lambda_{\mathrm{NDA}}$ is the UV cutoff.

The different limits on $M_{D}$ and $\Lambda_{\mathrm{NDA}}$ for $\delta=1$ are shown in fig. 4 . This figure extends the results of ref. [4] to the case of one extra dimension $(\delta=1)$. Notice that the constraint on $c_{\Upsilon}$ is the dominant one for large values of $\Lambda_{\mathrm{NDA}}$ and, in particular, it rules out the case of strongly-interacting gravity at the weak scale. For low values of $\Lambda_{\mathrm{NDA}}$, the dominant constraint comes from graviton emission and the dimension- 8 operator $\mathcal{T}$ does not add new information.

Finally, we comment on graviton contributions to low-energy processes, which are generally irrelevant for $\delta>1$, but have to be reconsidered for $\delta=1$. First consider the decay $K^{+} \rightarrow \pi^{+} G$, which contributes to the observable measured as $\operatorname{BR}\left(K^{+} \rightarrow \pi^{+} \bar{\nu} \nu\right)=\left(1.47_{-0.89}^{+1.30}\right) \times 10^{-10}$ [14]. The naïve estimate, $\operatorname{BR}\left(K^{+} \rightarrow \pi^{+} G\right) \sim\left(m_{K} / M_{D}\right)^{2+\delta}$, suggests that this process is interesting for $\delta=1$. However a careful analysis gives a different result. The part of the chiral Lagrangian bilinear in the charged $K$ and $\pi$ mesons is

$$
\mathscr{L}=a_{i j}\left(D_{\mu} \phi_{i}\right)^{\dagger}\left(D^{\mu} \phi_{j}\right)-m_{i}^{2} \phi_{i}^{\dagger} \phi_{i}
$$

Here $\phi=\left(\pi^{-}, K^{-}\right), m^{2}=\left(m_{\pi}^{2}, m_{K}^{2}\right), a_{11}=a_{22}=1, a_{12}=a_{21}=-\sqrt{2} G_{F} f_{\pi}^{2} g_{8} \sin \theta_{\mathrm{C}}$, and $\left|g_{8}\right|=5.1$ is the octet coupling. With a field redefinition, we can simultaneously diagonalize both the kinetic and mass terms. The graviton is coupled to the current $T_{\mu \nu}=g_{\mu \nu} \mathscr{L}-\left(\partial \mathscr{L} / \partial \partial^{\mu} \phi_{i}\right) \partial_{\nu} \phi_{i}$, which is also diagonal. Therefore (in contrast with axion emission), one finds no tree-level contribution to $K^{+} \rightarrow \pi^{+} G$ [15]. Loop effects exist but, given the LEP limits previously obtained, they are very small, since they are suppressed by a factor $\left(m_{K} / M_{5}\right)^{3} /(4 \pi)^{4} \sim\left(2 \mathrm{TeV} / M_{5}\right)^{3} 6 \times 10^{-16}$. The processes $(q \bar{q}) \rightarrow G \gamma$ are possible at tree level, but no interesting bounds can be extracted from $J / \psi$ or $\Upsilon$ decays [15].

\section{Graviton signals at the LHC}

Collider signatures of KK gravitons occur through real-graviton emission and virtual-graviton effects. Real-emission signals give clean signatures with jets or leptons plus missing energy, a class of signals very carefully studied in the framework of supersymmetry. A hard cut on missing energy removes QCD backgrounds very efficiently and, even for jet final states, leaves us mostly with $W$ and $Z$ boson production processes in the Standard Model. These background processes are fairly well understood theoretically and have small rates. In this section we will also explore virtual-graviton search strategies

\footnotetext{
"We thank G. Isidori for proposing these tests and for useful discussions.
} 
in detail. These signals come from tree-level virtual-graviton exchange (the dimension- $8 \mathcal{T}$ operator) and one-loop graviton contributions, which lead to the dimension- 6 axial-axial operator $\Upsilon$. Because virtual-graviton effects have no golden cut to efficiently suppress Standard Model backgrounds, our focus moves from the computation of the signal rates to the estimate of the error on the background processes.

We move beyond a one-parameter description in which one identifies the $D$ dimensional Planck scale $M_{D}$ with the cutoff $\Lambda$ of the theory. We apply a cutoff $\Lambda$ to the graviton-emission and virtualgraviton exchange rates, setting the partonic cross section to zero when $\hat{s}>\Lambda^{2}$ 开 This cutoff is important for the LHC because with a hadronic collider energy of $14 \mathrm{TeV}$ we expect to probe a wide range of energies. Separating the cutoff from the Planck mass allows us to investigate the behavior of the effective KK theory for LHC observables. Unless explicitly stated otherwise, we combine the statistical significance $S / \sqrt{B}$ and the systematical significance $S / \Delta B$ in quadrature and we require a $5 \sigma$ effect.

The same cutoff $\Lambda$ is used to regulate the ultraviolet divergences that appears in operators induced by virtual-graviton exchange. These divergences are expected to be removed by the ultraviolet completion of our effective KK theory. Because we do not know this ultraviolet completion, the dependence on the cutoff $\Lambda$ parametrizes our ignorance of ultraviolet effects.

Effects from virtual graviton exchange are described in terms of $M_{D}, \Lambda$ and $\delta$. The dependence is defined by the coefficients $c_{\mathcal{T}}$ and $c_{\Upsilon}$ of the dimension- 8 and dimension- 6 operators, obtained in modified naïve dimensional analysis (NDA) [4]

$$
c_{\mathcal{T}}=\left\{\begin{array}{cc}
\frac{\pi^{\delta / 2}}{\Gamma(\delta / 2)} \frac{\Lambda_{\mathrm{NDA}}^{\delta-2}}{M_{D}^{\delta+2}} & \text { for } \delta>2 \\
\frac{\pi}{M_{D}^{4}} \ln \frac{\Lambda_{\mathrm{NDA}}^{2}}{E^{2}} & \text { for } \delta=2
\end{array}, \quad c_{\Upsilon}=\frac{1}{16} \frac{\pi^{\delta-2}}{\Gamma^{2}(\delta / 2)}\left(\frac{\Lambda_{\mathrm{NDA}}^{\delta+1}}{M_{D}^{\delta+2}}\right)^{2}\right.
$$

where $E$ is a typical partonic energy of the process. The expressions for $\delta=1$ are given by eqs. (23) and $(26)$.

The ratio $\Lambda / M_{D}$ controls how strongly gravity is coupled, i.e. the relative importance of tree and loop effects. Even in string models of quantum gravity this remains an unknown parameter, controlled by the vev of the dilaton. The maximal value of $\Lambda / M_{D}$ corresponds to the case of strongly-coupled gravity, where all loops give comparable contributions. Naïve dimensional analysis estimates it to be [4]

$$
\Lambda \lesssim \Lambda_{S} \equiv\left[16 \pi^{\frac{4-\delta}{2}} \Gamma\left(\frac{\delta}{2}\right)\right]^{\frac{1}{2+\delta}} M_{D} .
$$

For real graviton emission, the dependence on the ultraviolet cutoff $\Lambda$ measures the fraction of the signal coming from different regions of the KK mass spectrum, while the matrix element itself is independent of $\Lambda$. Again, we limit the parton energies to $\sqrt{\hat{s}}<\Lambda \lesssim \Lambda_{S}$.

We stress that care should be applied when different signals are compared, since the cutoff $\Lambda$ will be in general different for different processes: order one factors can be fixed only after understanding quantum gravity.

\subsection{Graviton emission}

Two well-suited search channels for real-graviton emission are a single hard jet plus missing energy [3] and Drell-Yan lepton pairs plus missing energy [17]. The latter is a particularly clean channel, but

\footnotetext{
${ }^{\|}$Ref. [16] suppressed the cross sections in a slightly different way, by adding in a factor $\Lambda^{4} / \hat{s}^{2}$ for $\hat{s}>\Lambda^{2}$. Our results and those in 16] are in good agreement.
} 


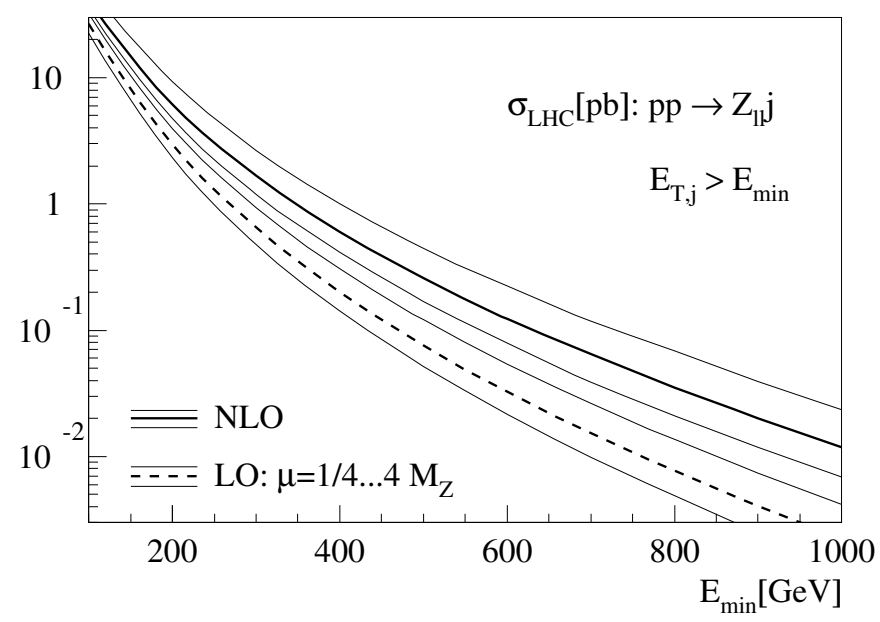

Figure 5: The leading order and NLO cross sections for the process $p p \rightarrow Z+$ jet with a subsequent $Z$ decay into electrons or muons [18]. The factorization and renormalization scales are set equal and varied around a common central value. The only cut applied is the shown minimum cut on the transverse jet momentum.

its reach has shown to be somewhat less than the single jet counterpart's. We therefore concentrate on the process $p p \rightarrow \mathrm{G}+$ jet.

We perform a parton-level analysis, including the dominant irreducible background $p p \rightarrow Z+$ jet where the $Z$ decays to neutrinos. Apart from a basic acceptance cut for the jet, $\left|\eta_{j}\right|<4.5$, we apply a variable transverse momentum cut: $p_{T, j}>\Lambda / 4$ for $\Lambda<4 \mathrm{TeV}$ and $p_{T, j}>1 \mathrm{TeV}$ otherwise. To first approximation the signal and the dominant background processes will lead to a back-to-back signature of the jet with the invisible KK graviton or $Z$ boson, respectively. Therefore, there is no need to apply a hard missing-energy cut beyond the triggering requirements - for each event we expect $\not_{T} \sim p_{T, j}$. A minimum $\not_{T}$ cut could be added, but would only increase the uncertainty because of the $\mathscr{H}_{T}$ smearing of the detector and the appropriate calibration. The second largest background arises from $p p \rightarrow W+$ jet, where we lose the lepton from the $W$ decay because it is too soft or too far forward. Even though the rate before acceptance cuts is much larger than the $Z+$ jet rate, after cuts it only leads to a $\sim 10 \%$ correction to the $Z+$ jet background.

The nature of the graviton signal does not permit any kind of simple side-bin analysis. All information is encoded in the hard $p_{T, j}$ spectrum of the signal plus the $Z+$ jet background. The NLO correction to the backgrounds $p p \rightarrow Z / W+$ jet are known [18]. We show the leading order as well as the NLO cross sections as a function of the $p_{T, j}^{\min }$ cut in fig. 5 . If we do not apply any cut, we obtain a $K$ factor of 1.6 and a remaining NLO scale variation of the order of $25 \%$. For a large $p_{T, j}$ cut the relative correction increases to $K \sim 2$ with a remaining NLO scale uncertainty of almost $100 \%$. This does not even take into account the fact that we have two scales present in the process, namely the weak boson mass $m_{Z}$ and the transverse momentum of the jet - in principle we would have to resum large logarithms $\log p_{T, j} / M_{Z}$. Therefore we can immediately conclude that trying to extract the KK signal out of the $Z+$ jet background using the theoretical prediction of the $p_{T, j}$ spectrum of the $Z+$ jet final state will have to cope with very large systematic errors and it is therefore pointless.

Instead, we normalize the $Z+$ jet rate using $Z$ decays to electrons and muons, as suggested in ref. [3. Since this final state can be fully reconstructed, we can even mimic signal cuts and look for effects from large logarithms induced by the $p_{T, j}$ cut in the actual analysis. The remaining statistical uncertainty on the background measurement can be estimated from fig. 5 . Using only $10 \mathrm{fb}^{-1}$ of data 

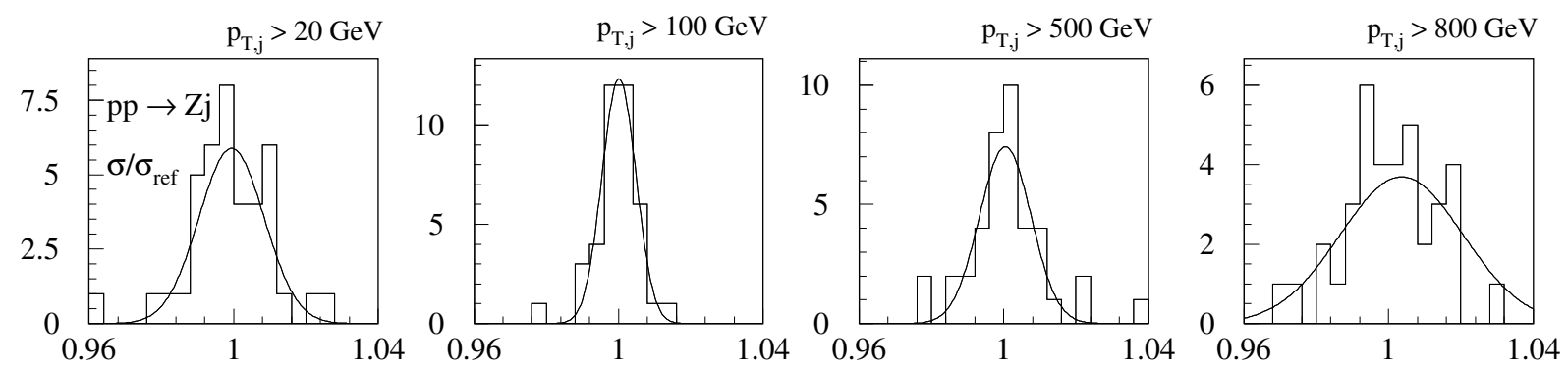

Figure 6: The range of $Z+$ jet background cross sections, due to the uncertainty in the parton densities. The histograms show the deviation $\sigma / \sigma_{\mathrm{Cteq} 6.1 \mathrm{M}}$. The set of 40 parton densities included in the histograms is described in ref. [19].

and a $p_{T, j}$ cut of $600 \mathrm{GeV}$, we are left with $S=1000$ events for one lepton flavor, which gives us a statistical uncertainty of $1 / \sqrt{S} \sim 3 \%$ per lepton flavor. Combining both the electron and the muon final state it seems to be conservative to assume a systematic uncertainty of $5 \%$ on the prediction of the $Z+$ jet background cross section including detector effects.

The $W+$ jet background cannot be reconstructed, because the three-quark final state with a hadronic $W$ decay will be dominated by the large QCD three-jet continuum. However, the NLO corrections scale with the $Z+$ jet production process; i.e. the $W+$ jet rate can be estimated from the measured $Z+$ jet rate. Since the $W+$ jet rate gives a contribution to the total background of at most $10 \%$, and is affected by an uncertainty of less than $20 \%$, we can essentially neglect this contribution, which is taken into account in the $5 \%$ quoted uncertainty.

For completeness, we study the uncertainty caused by the parton densities. This source of uncertainty can be possibly reduced through a complete two-loop analysis with HERA and hadron collider jet data, but it should not just be neglected. For different $p_{T, j}$ cuts we show histograms using a consistent set of different Cteq6.1 parton densities [19] in fig. 6. As expected, we observe a very narrow distribution if we require $p_{T, j}>100 \mathrm{GeV}$, pushing the parton momentum fraction into the well determined region $x \sim 1 / 100$. In the region of larger transverse jet momenta, where we can observe the graviton radiation signal, the probed $x$ values become larger, but the systematic uncertainty on the cross section prediction inherited from uncertainties in the parton density input does not grow beyond $2 \%$, the maximum width we observe in fig. 6. This small uncertainty can be traced back to the parton kinematics: while the uncertainty on the gluon densities for $x>0.5$ is indeed very large, it stays small for valence quarks [19]. We include at maximum one gluon in the initial state. When the gluon luminosity drops sharply for large $x$ values, the mixed $q g$ initial state will be pushed to asymmetric $x_{g} \ll x_{q}$ configurations, which do not suffer from large uncertainties. Last but not least, this theoretical uncertainty is of no concern if we measure the $Z+$ jet background, but even if we were to combine the measured background rate and its theoretical prediction the parton densities would be no reason to worry.

In fig. 7 we show the discovery reach for graviton plus jet production at the LHC, assuming an integrated luminosity of $10 \mathrm{fb}^{-1}$ and $100 \mathrm{fb}^{-1}$. The area to the right and below the lines will lead to a $5 \sigma$ signal with combined systematical and statistical errors. Large values of $M_{D}$ lead to a direct suppression of the graviton coupling and thereby a suppression of the signal rate. Smaller cutoffs remove a larger fraction of the graviton mass spectrum we integrate over.

The plateau reached by the curves at large $\Lambda$ indicates the region where the signal becomes independent of the ultraviolet cutoff. If the onset of the plateau starts when $\Lambda$ is in the perturbative regime $\left(\Lambda<\Lambda_{S}\right)$, the use of the effective theory, obtained by linearizing Einstein gravity, is well 


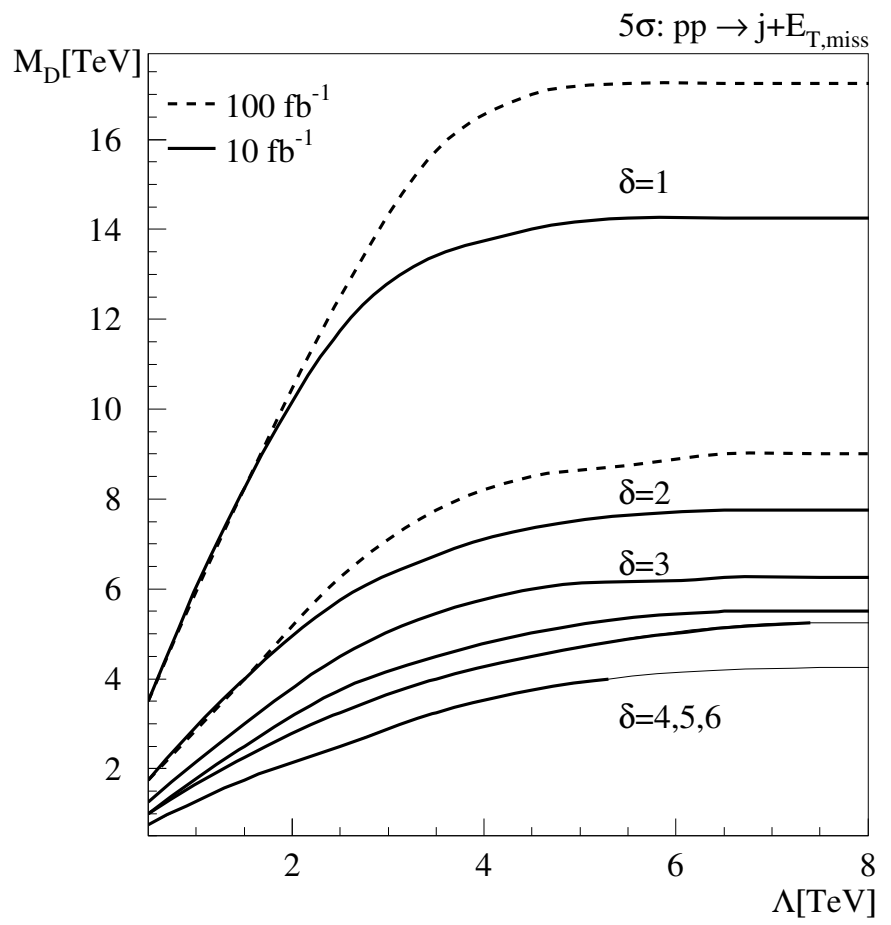

Figure 7: The LHC reach in the search for graviton emission. The number of extra dimensions $\delta$ is varied from 1 to 6 , in the lines from top to bottom. The switch from thick to thin lines indicates the end of the region $\Lambda<\Lambda_{S}$.

justified (for the values of $M_{D}$ probed). In this case, the contribution to the signal coming from very energetic gravitons is strongly suppressed by parton luminosity. On the other hand, if the plateau is reached only for $\Lambda>\Lambda_{S}$, the signal is sensitive to the unknown physics from the ultraviolet completion of the effective theory. This happens for $\delta \gtrsim 5$, as shown in fig. 7. Over some fraction of the discovery contour shown in fig. 7 we find $M_{D} \sim \Lambda \sim \sqrt{\hat{s}}$. While the one-graviton production cross section remains finite even for large cutoff values, the complete $\not_{T}$ signal will consist of an uncalculable increasing fraction of multi-graviton emission.

For a smaller number of extra dimensions, the graviton mass spectrum is dominated by lighter modes, since the graviton mass distribution is proportional to $m^{\delta-1} d m$ (and, in particular, becomes flat for $\delta=1$ ). This means that, for small $\delta$, the cutoff will have a less dramatic effect: the transition to the plateau is more abrupt and the discovery reach becomes independent of $\Lambda$ already for $\Lambda \ll \Lambda_{S}$. Moreover, since the cross section is proportional to $M_{D}^{-2-\delta}$, we can probe much larger values of $M_{D}$ for a small number of extra dimensions. For larger values of $\delta$, the signal is dominated by gravitons with the largest possible energy, and therefore the reach on $M_{D}$ critically depends on $\Lambda$, as shown in fig. 7 .

We recall that the curves in fig. 7 show the upper limit on $M_{D}$ that can be reached by LHC. The successful theoretical description of the LHC reach is based on the separation of the two scales $\sqrt{\hat{s}}<M_{D}$, which works very well for the maximum $M_{D}$ values which can be probed. A lower bound on the range of $M_{D}$ which can be probed is given by the theoretical requirement $\Lambda<\Lambda_{S}$ and the condition that $\Lambda$ has to be larger than the typical partonic collider energy, thereby allowing for the observed saturation behavior. For smaller values of $M_{D}$ the requirement that gravity cannot become strongly interacting can lead a theoretical uncertainty $d \sigma\left(M_{D}, \delta ; \Lambda\right) / d \Lambda \neq 0$ that has to be properly taken into account. Looking at the different values of $\delta$ we conclude that LHC searches for KK emission 
become more probing and less theoretically uncertain the smaller the number of extra dimensions.

The improvement of the LHC reach with a luminosity increase is linked to either a dominating systematical or statistical error: for small cutoff values $\Lambda$, the transverse momentum cut is small as well, leaving us with a large background rate. The systematical error on this large background rate determines the reach, so a luminosity increase will only slightly improve the reach. Taking into account detector effects, a purely high-luminosity sample is likely to be inferior to its low-luminosity counterpart. Once we probe larger values of $\Lambda$, which is possible for a small number of extra dimensions, the background suppression cut tightens and a luminosity increase does have a sizeable effect, as we can see in the $\delta=1,2$ cases in fig. 7 .

We have confronted the real graviton emission with the Standard Model process $p p \rightarrow Z+$ jet, but new backgrounds can arise from the unknown dynamics at the cutoff scale. Although tree-level graviton exchange does not directly contribute to the dominant parton subprocess $q g \rightarrow q Z$, in general there could be other effective operators involving neutrinos or $Z$ bosons, which can affect our background measurement. While we know from LEP that on the $Z$ pole virtual graviton exchange cannot have a large effect, unknown neutrino operators could mimic a KK mediated deviation from the Standard Model $p p \rightarrow Z+$ jet prediction.

\subsection{Tree-level virtual graviton exchange}

There are three most promising candidate channels to study tree-level virtual graviton effects: DrellYan lepton pairs, photon pairs and two jets. The dimension- 8 operator $\mathcal{T}$ induced by tree-level graviton exchange can interfere with Standard Model contributions for $\delta>1$ and with the elastic contribution for $\delta=1$. Therefore, it can lead to effects suppressed by only one power of $\mathcal{S} \sim M_{D}^{-2-\delta}$. Because we are dealing with interfering amplitudes, there will be no golden cut to distinguish signal and backgrounds; at most we expect significant changes in some distribution. Instead of the transverse momentum distribution which we have utilized for the real emission, we now study the invariant mass of the final state leptons or photons or jets to measure a deviation from the Standard Model behavior. In contrast to the real emission case, there is no independent background measurement, but the Drell-Yan $M_{\ell \ell}$ spectrum can at least be normalized at the low end just above the $Z$ pole, where the heavy KK states should not contribute.

In earlier works the reaches of the two lepton and the two photon channels have been found to be comparable [20, 21]. The diphoton processes, however, suffers from several disadvantages: the total rate is only known at NLO [22], with an estimated error of about 20-30\%. A normalization of the invariant mass distribution in the small $M_{\gamma \gamma}$ region might suffer from soft-photon radiation on the one hand, and its extrapolation is affected by large logarithms on the other hand. Finally the error due to QCD uncertainties of misidentified photons will be at least of the order of $10 \%$ to $20 \%$ for the extracted photon rate, well above the estimated error for the Drell-Yan process [22].

Again, we only require basic cuts for the leptons $\left|\eta_{\ell}\right|<2.5$ and $p_{T, \ell}>50 \mathrm{GeV}$, plus a variable minimum cut $M_{\ell \ell}>\Lambda / 2$ for $\Lambda<4 \mathrm{TeV}$ and $M_{\ell \ell}>2 \mathrm{TeV}$ otherwise. The $5 \sigma$ reach of this channel is illustrated in fig. 8. We start by showing our results, in the left panel of the figure, by using the model-independent parameters $M_{\text {eff }}$ (where $c_{\mathcal{T}} \equiv 8 / M_{\text {eff }}^{4}$ ) and $\Lambda$, the ultraviolet cutoff. In this case, $\Lambda$ corresponds to the maximum allowed value of the two-lepton invariant mass $M_{\ell \ell}$.

In contrast to the background, the KK signal involves two parton subprocesses: the usual DrellYan type diagram with an $s$ channel KK exchange, and a gluon initiated process. The latter will have a large effect on the total rate at the LHC, but the $M_{\ell \ell}$ cut selects the large parton $x$ regime where initial state quarks dominate (see left panel of fig. 8). The interference between KK and Standard Model contributions to the Drell-Yan channel is strongly suppressed by a combination of couplings 

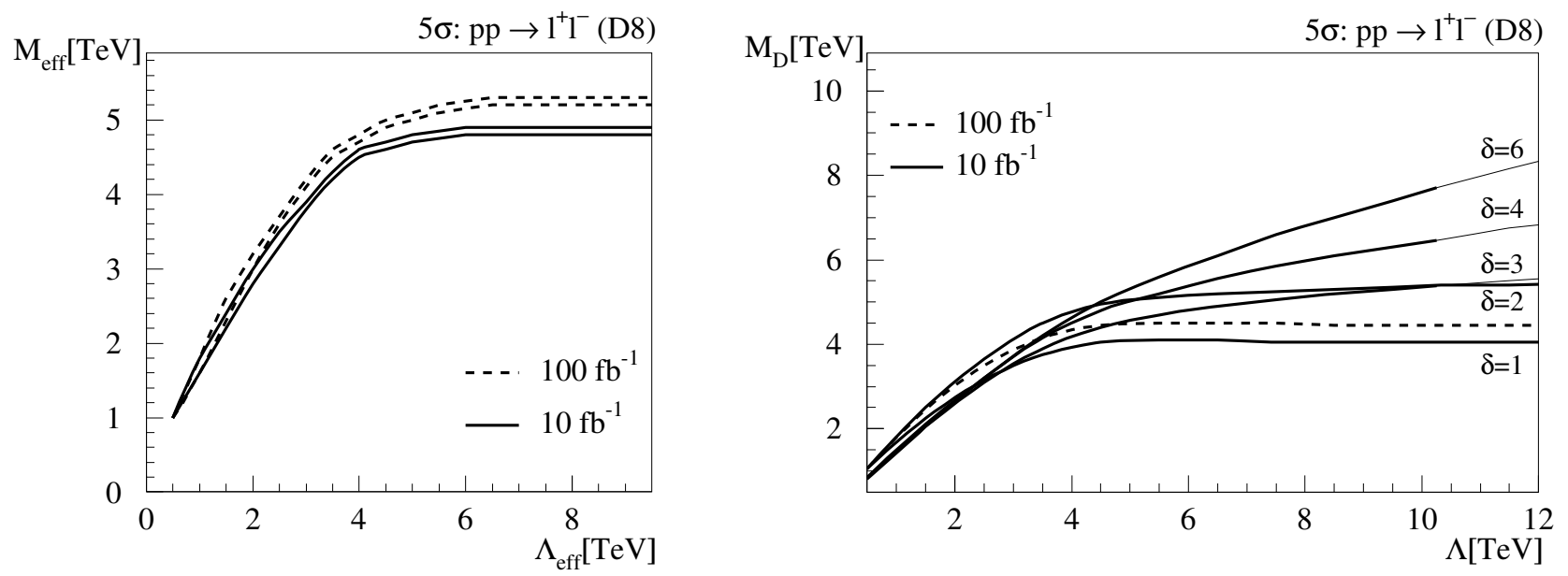

Figure 8: The LHC reach in the search for virtual graviton effects through the dimension-8 operator $\mathcal{T}$ in Drell-Yan lepton pair production. Left: the result in terms of the coefficient $c_{\mathcal{T}}$ of the dimension-8 operator, with $c_{\mathcal{T}}= \pm 8 / M_{\mathrm{eff}}^{4}$ (the sign does not affect the result). The reach is shown for the $q \bar{q}$ initial state only (lower $M_{\text {eff }}$ ) and including the gg initial state (higher $M_{\text {eff }}$ ). The cutoff $\Lambda_{\text {eff }}$ acts on the partonic collider energy and defines the minimum $M_{\ell \ell}$ cut exactly like $\Lambda$ in the extra-dimensions parametrization. Right: the same result in terms of the extra-dimension parameters $\delta, \Lambda$, and $M_{D}$. The switch from thick to thin lines indicates the end of the region $\Lambda<\Lambda_{S}\left(M_{D}\right)$.

and phase space factors when we integrate over the entire phase space. Therefore, the rate for the KK effects is not proportional to $c_{\mathcal{T}}$, but to $c_{\mathcal{T}}^{2}$. Indeed the curves for positive and negative values of $c_{\mathcal{T}}$ in the left panel of fig. 8 are nearly identical. Taking into account only the interference term would reduce the LHC reach to $M_{\text {eff }}<2 \mathrm{TeV}$. We note, however, that it might still be possible to enhance the reach applying a more sophisticated set of cuts to increase the interference effects. As long as $\Lambda$ is larger than about $4 \mathrm{TeV}$, the reach shown in the left panel of fig. 8 is independent of $\Lambda$, since the condition $M_{\ell \ell}<\Lambda$ does not reduce the signal.

Next, we show in the right panel of fig. 8 the same result expressing $c_{\mathcal{T}}$ in terms of $\Lambda$ and $M_{D}$, as given in eq. (28). For $\delta>2$, the LHC reach on $M_{D}$ grows with $\Lambda$, as an effect of this particular parametrization. The form of $\mathcal{S}$ will lead to a scaling $M_{D}^{\max } \propto \Lambda^{(\delta-2) /(\delta+2)}$ for $\Lambda \gtrsim 1 \mathrm{TeV}$. The growth is limited by the requirement $\Lambda<\Lambda_{S}$ which implies $\Lambda<(\sqrt{2} \pi)^{1 / 2} M_{\mathrm{eff}}^{\max } \simeq 10 \mathrm{TeV}$, for any $\delta>2$, where $M_{\text {eff }}^{\max }$ is the reach on $M_{\text {eff }}$, shown in fig. 8. Because the interference between KK and Standard Model contributions is suppressed, the numerical impact of a shift $\Lambda_{S}=M_{D} \rightarrow 4 M_{D}$ on the total cross section could be as large as a factor $4^{2(\delta-2)}$. For $\delta=6$ we find a change of the cross section by more than a factor $10^{5}$ for fixed $M_{D}=4 \mathrm{TeV}$ and a fixed kinematic cut $M_{\ell \ell}>1 \mathrm{TeV}$. The total cross section, however, is extremely sensitive to changes in $\Lambda$ and also in $M_{D}$. If we translate the factor $4^{2(\delta-2)}$ into a compensating shift in $M_{D}$ we only get a shift by a factor $(4)^{(\delta-2) /(\delta+2)}$, which for $\delta=6$ means $M_{D} \rightarrow 2 M_{D}$.

For $\delta=2$ the cutoff dependence of $c_{\mathcal{T}}$ is only logarithmic, and $c_{\mathcal{T}}$ is finite for $\delta=1$. In fig. 8 we see that, for these two cases, the LHC reach is practically independent of $\Lambda$, as soon as $\Lambda>4 \mathrm{TeV}$, when the condition $M_{\ell \ell}<\Lambda$ no longer affects the signal. Therefore, for small $\delta$ (and especially for $\delta=1$ ), the coefficient is less sensitive to the ultraviolet behavior of the theory, here parametrized by $\Lambda$. This means that the search for tree-level virtual-graviton effects, although theoretically more under control, covers a smaller portion of the $M_{D^{-}} \Lambda$ region, as the value of $\delta$ is decreased. This is in contrast to real-graviton emission, where the reach is enhanced for small $\delta$.

The two-jet channel is of course tempting at the LHC, just because of its large rate. However, this 


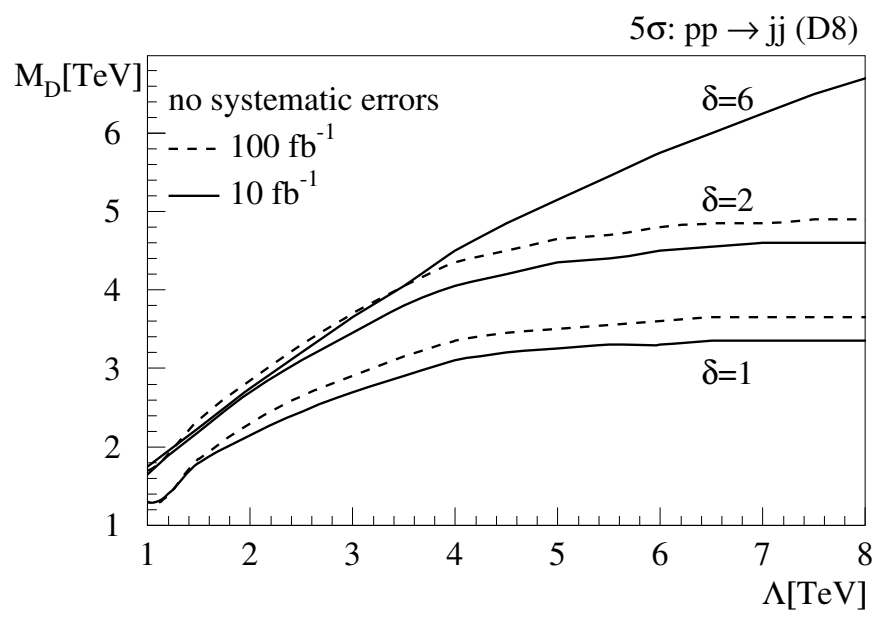

Figure 9: The LHC reach in the search for tree-level virtual-graviton effects through the dimension-8 operator $\mathcal{T}$ in two-jet process. For illustrative purposes, the systematic errors are set to zero, even though they will dominate and even nullify the reach.

rate also comes with a large uncertainty, not only on the total rate but also on distributions. The invariant mass spectrum for example is a likely candidate to measure parton distribution functions, which means that even if we knew its normalization (which we do not know) it would be hard to extract any information from it. In fig. 9 we show an example for discovery reach of the LHC looking for dimension-8 virtual graviton effects for two jet production in the limit of zero systematical uncertainty. We see that the reach is similar to the corresponding Drell-Yan process; however, we find $S / B$ well below $10^{-2}$, which means that we do not even have to estimate a systematical error on the QCD two jet production - any reasonable number will return a discovery potential worse than in the lepton pair channel.

\subsection{One-loop virtual graviton exchange}

Graviton loops induce a dimension-6 operator $\Upsilon$ [4, 23]. We perform the same analysis of the DrellYan lepton pairs at the LHC as for the dimension- 8 operator $\mathcal{T}$. First, we determine the LHC reach in terms of the parametrization $c_{\Upsilon}= \pm 4 \pi / M_{\text {eff }}^{2}$. Because of the cancellation between interference effects and the dimension-6 operator squared, a positive sign $c \Upsilon$ has a reduced effect on the Drell-Yan cross section. From fig. 10 we observe that the cutoff dependence disappears for $\Lambda>4 \mathrm{TeV}$, which is only slightly above the typical $M_{\ell \ell}$ values at the LHC and well below the maximum values $M_{\text {eff }} \sim 25-$ $40 \mathrm{TeV}$. The two scales are well separated, and the result is fairly independent of the cutoff. We can translate the maximum range in terms of the dimension- 8 operator $c_{\mathcal{T}}^{2}=\left(8 / M_{\text {eff }}^{4}\right)^{2}$ into the range for the dimension-6 operator $c_{\Upsilon}=4 \pi / M_{\text {eff }}^{2}$, assuming the typical partonic collider energy is $M_{\ell \ell} \sim 2 \mathrm{TeV}$.

As a second step we interpret the same result in terms of the extra-dimensional effective theory, using the definition of the dimension-6 operator coefficient $c_{\Upsilon}$ in eq. (28). The dependence on $\Lambda$ of $c_{\Upsilon} \sim \Lambda^{2 \delta+2} / M_{D}^{2 \delta+4}$ is steeper than for the real emission and the dimension-8 cases. Moreover, comparing the two parametrizations of the dimension-6 operator for $M_{D}=\Lambda=M_{\text {eff }}$ we find a considerably smaller prefactor for $c_{\Upsilon}$ for the extra dimension ansatz. Correspondingly, the $5 \sigma$ discovery curves in fig. 10, shown for positive $c_{\Upsilon}$, only barely cross the diagonal $\Lambda<M_{D}$. In contrast to the dimension-8 operator the dimension- 6 analysis will have basically no reach at the LHC if we limit ourselves to $\Lambda<M_{D}$, but surpasses the dimension-8 operator for $M_{D}<\Lambda<\Lambda_{S}$. This is consistent 

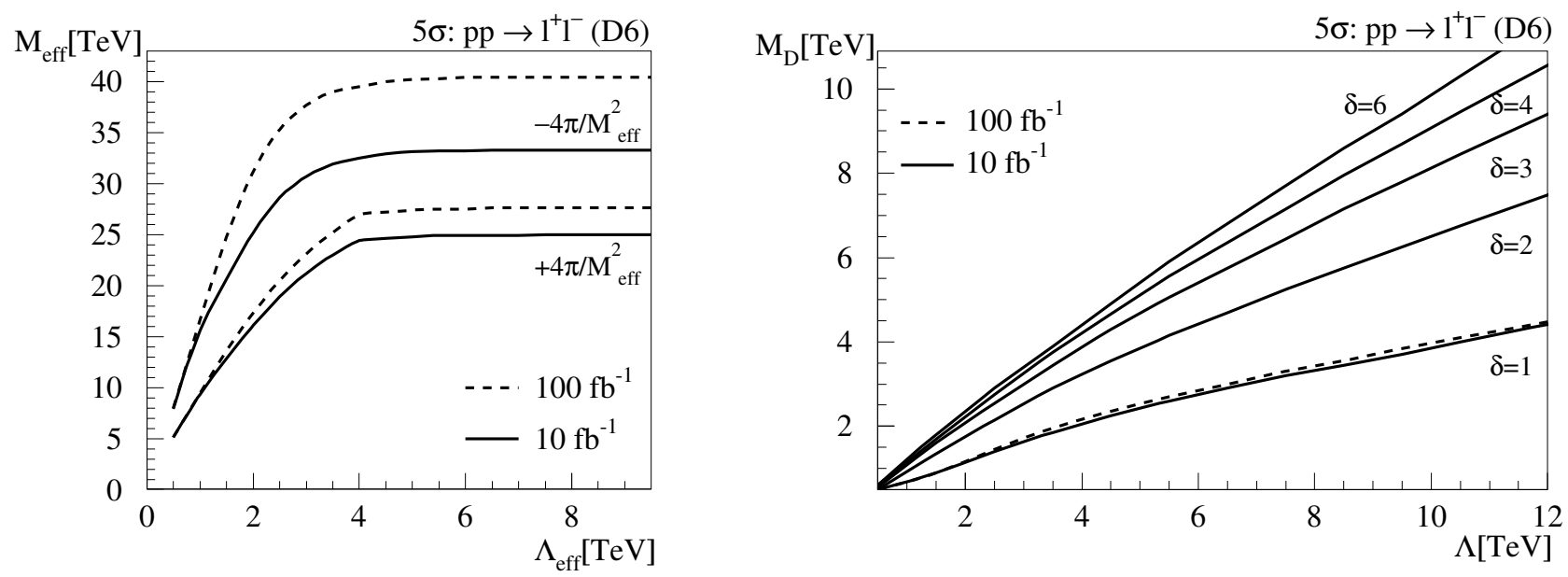

Figure 10: The LHC reach in the search for graviton-loop effects through the dimension- 6 operator $\Upsilon$ in Drell-Yan lepton-pair production. The cutoff $\Lambda_{\mathrm{eff}}$ acts on the partonic collider energy and defines the minimum $M_{\ell \ell}$ cut exactly like $\Lambda$ in the extra-dimension parametrization. Left: the results in terms of the parametrization $c_{\Upsilon}= \pm 4 \pi / M_{\mathrm{eff}}^{2}$. Right: the reach in terms of the parametrization in eq. (28), for a positive value of $c_{\Upsilon}$.

with the notion that loop effects become overwhelming when $\Lambda$ is large, and the effective gravitational coupling becomes sufficiently strong.

The reach on $M_{D}$ shown in fig. 10 grows with $\Lambda$ almost linearly, $M_{D}^{\max } \propto \Lambda^{(\delta+1) /(\delta+2)}$, until it is overcome by the condition $\Lambda<\Lambda_{S}$. This happens for $\Lambda=2 \pi^{1 / 2} M_{\mathrm{eff}}^{\max } \sim 89 \mathrm{TeV}$, for any $\delta$. This means that searches for the operator $\Upsilon$ can very efficiently cover the case of strong gravity $\left(\Lambda \gtrsim M_{D}\right)$ in a large range of $M_{D}$ values, namely up to $M_{D}^{\max }=(16,33,47,56,62,66) \mathrm{TeV}$ for $\delta=(1, \ldots, 6)$. This typical scale of $M_{D}^{\max }$ is set by the maximum value of $M_{\text {eff }}$ which we can probe at the LHC. This corresponds to the hierarchy of scales in the dimension- 8 case, where the typical scale is set by $M_{\text {eff }} \sim 5 \mathrm{TeV}$, the reach in $M_{D}$ is somewhat larger (depending on $\delta$ ), and $\Lambda$ is fixed by the condition $\Lambda<\Lambda_{S}\left(M_{D}\right)$. The collider energy as a scale has no impact once $s / M_{\text {eff }}^{2}$ fixes the reach in $M_{\text {eff }}$.

\section{Conclusions}

We have reconsidered the physics of KK graviton excitations in theories with $\delta$ large extra dimensions. The high-energy and low-energy ends of the KK spectrum lead to different effects.

- The heaviest KK modes affect high-energy experiments in a way that does not depend on the detailed shape of the compactification. However, while graviton emission is a truly modelindependent test, virtual-graviton exchange for $\delta \geq 2$ cannot be fully computed without knowledge of the UV completion.

- The lightest KK modes affect Newtonian gravity at measurable distances and astrophysical processes in a fully computable way that however depends on the detailed shape of the extra dimensions. In models with $\delta$ flat extra dimensions with equal radii, the KK masses are $m \sim$ $M_{D}\left(M_{D} / M_{\mathrm{Pl}}\right)^{1 / \delta}$, leading to conflict with observations for $\delta \leq 3$ and $M_{D}<3 \mathrm{TeV}$.

Many previous works studied high-energy signals of $\delta=2,3$ extra dimensions, tacitly assuming the existence of some appropriate non-minimal shape that removes the light KK modes. The case $\delta=1$ was ignored, maybe because it is less trivial to imagine an appropriate non-minimal shape for a one- 
dimensional extra dimension, or maybe just because of psychological reasons: in the flat limit the case $\delta=1$ contradicts even ordinary experience.

In section 2 we discussed how making the long extra dimension slightly warped introduces an extra mass $\mu$ to the spectrum of KK excitations that allows to make all states heavier than about $50 \mathrm{MeV}$, avoiding problems with observations. Warping also makes each KK more strongly coupled: the combined effect of these two factors leaves unaffected high-energy experiments which cannot resolve the individual KK modes.

The explicit model motivates our later study of high-energy signals, which do not depend on the specific mechanism introduced to cure low-energy problems. In section 3 we studied collider signals of a single gravitational large extra dimension, findings a few peculiarities.

1) Unlike $\delta \geq 2$, for $\delta=1$ the graviton-emission cross sections are not dominated by events with the largest kinematically allowed graviton energy. LEP measurements of $e^{+} e^{-} \rightarrow \gamma G$ events with large $E_{\gamma} \gtrsim M_{Z}$ give the constraint $M_{D} \gtrsim 2.4 \mathrm{TeV}$ on the 5-dimensional Planck mass (normalized as in ref. [3]). Furthermore, unpublished $e^{+} e^{-} \rightarrow Z G$ data might give a competitive test.

2) For $\delta \geq 2$ tree-level virtual graviton exchange gives the dimension- 8 operator $\mathcal{T}$ of eq. (19) which has an uncomputable UV-divergent coefficient [3]. For $\delta=1$ tree-level virtual graviton exchange gives a dimension-7 fully computable amplitude, which interferes with the SM amplitude only in elastic channels. The dominant effect at LEP is an increase of the $e^{+} e^{-} \rightarrow e^{+} e^{-}$cross section, with a peculiar scattering-angle dependence. The present constraint is $M_{D} \gtrsim 2.4 \mathrm{TeV}$.

3) One loop graviton exchange gives the dimension-6 operator $\Upsilon$ of eq. (25); its coefficient is UVdivergent coefficient for any $\delta$, including $\delta=1$.

Finally we reconsidered how the sensitivity of LHC searches to gravitons in $\delta$ large extra dimensions depends on the cut-off $\Lambda$ of the theory. Previous analyses assumed $\Lambda=M_{D}$, which fixes in an arbitrary way the relative merit of the different signals discussed above. In appendix A we give the cross sections of graviton-induced hadronic processes relevant for LHC studies (with finding that do not fully agree with previous results valid for $\delta \geq 2$ ).

Searches for real-graviton emission, tree-level graviton exchange, and graviton-loop effects give complementary information. For graviton emission, we have focused on the jet and missing energy channel. The case $\delta=1$ is particularly interesting, since LHC can probe values of $M_{D}$ up to $14 \mathrm{TeV}$ (for an integrated luminosity of $10 \mathrm{fb}^{-1}$ ) or $17 \mathrm{TeV}$ (for $100 \mathrm{fb}^{-1}$ ). The reach rapidly deteriorates as $\delta$ is increased, becoming very sensitive to the cut-off $\Lambda$ and therefore to the unknown ultraviolet behavior of the theory.

Tree-level graviton exchange is best studied at the LHC in the Drell-Yan channel, which can probe regions of the $\left(M_{D}, \Lambda\right)$ plane not accessible to other searches. The case $\delta=1$ allows a prediction of the rate, which is independent of the ultraviolet cutoff, with the result that graviton emission is a more sensitive probe than tree-level graviton exchange.

Graviton-loop effects can dominate over tree-level exchange because they induce operators with lower dimensionality. In the regime where gravity can become strong $\left(\Lambda \gtrsim M_{D}\right)$, the search for the effective operator $\Upsilon$ in the Drell-Yan channel provide the most efficient probe of theories with multi-dimensional gravity.

Acknowledgments We thank G. Isidori, S. Mele and R. Rattazzi for interesting discussions. We thank I. De Bonis, R. Tenchini and P. Wells for providing and explaining us the results on $e \bar{e} \rightarrow e \bar{e}$ data. 


\section{A Cross sections of graviton processes}

Here we list all cross sections necessary for studying the signals generated by tree-level exchange of gravitons in $\delta \geq 1$ extra dimensions, with the following final states: $f \bar{f}$ and $\gamma \gamma$ at electron-positron colliders, and jet jet, $\gamma \gamma, \ell \bar{\ell}$ at hadron colliders. It is useful to define

$$
G(s, t) \equiv \frac{s^{4}+10 s^{3} t+42 s^{2} t^{2}+64 s t^{3}+32 t^{4}}{4} .
$$

Here $s, t, u$ are the usual Mandelstam variables, related by $s+t+u=0$. All our cross section are normalized such that the integration range is $-s<t<0$, even when there are two identical particles in the final state.

The effective graviton propagator $\mathcal{S}$ in $\delta$ extra dimensions is defined in eq. (21). For $\delta>2$ the uncomputable UV divergent contribution dominates and our expressions can be simplified setting $\mathcal{S}(s)=\mathcal{S}(t)=\mathcal{S}(u)=\mathcal{S}$, which is the limit studied in the previous literature**

\section{Electron positron $\rightarrow$ fermion antifermion.}

$$
\begin{aligned}
\frac{d \sigma}{d t}\left(e^{+} e^{-} \leftrightarrow f \bar{f}\right)= & \frac{u^{2}\left(\left|G^{L L}\right|^{2}+\left|G^{R R}\right|^{2}\right)+t^{2}\left(\left|G_{s}^{L R}\right|^{2}+\left|G_{s}^{R L}\right|^{2}\right)+s^{2}\left(\left|G_{t}^{L R}\right|^{2}+\left|G_{t}^{R L}\right|^{2}\right)}{16 \pi s^{2}} \\
& +\frac{1}{128 \pi s^{2}}\left[|\mathcal{S}(s)|^{2} G(s, t)+|\mathcal{S}(t)|^{2} G(t, s)\right. \\
& +2 \operatorname{Re} \mathcal{S}(s)^{*}\left(\left(G_{s}^{L R}+G_{s}^{R L}\right) t^{2}(3 s+4 t)+\left(G^{L L}+G^{R R}\right)(s+4 t) u^{2}\right) \\
& +2 \operatorname{Re} \mathcal{S}(t)^{*}\left(\left(G_{t}^{L R}+G_{t}^{R L}\right) s^{2}(4 s+3 t)+\left(G^{L L}+G^{R R}\right)(4 s+t) u^{2}\right) \delta_{e f} \\
& \left.+\frac{\operatorname{Re} \mathcal{S}(s) \mathcal{S}(t)^{*}}{2}(4 s+t)(4 t+s) u^{2} \delta_{e f}\right]
\end{aligned}
$$

where $\delta_{e f}=1$ if $f=e$ and $\delta_{e f}=0$ otherwise. If $f$ is a quark $d \sigma(e \bar{e} \rightarrow f \bar{f})$ must be multiplied by $N_{f}=3$ while $d \sigma(f \bar{f} \rightarrow e \bar{e})$ must be divided by $N_{f}$. The factors

$$
G_{s}^{A B} \equiv \sum_{V=\gamma, Z, W} \frac{g_{A}(V \rightarrow \ell \bar{\ell}) g_{B}(V \rightarrow f \bar{f})}{s-M_{V}^{2}+i M_{V} \Gamma_{V}}, \quad G_{t}^{A B} \equiv \sum_{V=\gamma, Z, W} \frac{g_{A}(V \rightarrow f \bar{\ell}) g_{B}(V \rightarrow \ell \bar{f})}{t-M_{V}^{2}}
$$

$(A, B=\{L, R\})$ are the propagators of electroweak SM gauge bosons, and $G^{L L}=G_{s}^{L L}+G_{t}^{L L}$, $G^{R R}=G_{s}^{R R}+G_{t}^{R R}$. If $f \neq e, \nu_{e}$ there is no $t$-channel contribution, $G_{t}^{A B}=0$. In the case of Bhabha scattering $e^{+} e^{-} \rightarrow e^{+} e^{-}$one has

$$
G_{x}^{A B}=\frac{e^{2}}{x}+\frac{g_{2}^{2} g_{A e} g_{B e} / \cos ^{2} \theta_{\mathrm{W}}}{x-M_{Z}^{2}+i \Gamma_{Z} M_{Z}} \quad x=\{s, t\}
$$

with $g_{L e}=\sin ^{2} \theta_{\mathrm{W}}-1 / 2$ and $g_{R e}=\sin ^{2} \theta_{\mathrm{W}}$ 闯

** Our results agree with ref. [3, but we find the following discrepancies with the cross sections for the hadronic processes computed in ref. 24]. Their parameter $F / M_{S}^{4}$ corresponds to our $-\mathcal{S} / 8 \pi$. In $q q \rightarrow q q, q \bar{q} \rightarrow q \bar{q}$ and $\bar{q} \bar{q} \rightarrow \bar{q} \bar{q}$ scatterings our results would agree using instead $F / M_{S}^{4} \leftrightarrow \mathcal{S} / 4 \pi$. In $g g \rightarrow q \bar{q}$ scattering we would agree for $F / M_{S}^{4} \leftrightarrow$ $-\mathcal{S} / 4 \pi$. The pure graviton contribution to $g g \rightarrow g g$ is 4 times bigger than in ref. 24].

${ }^{\dagger \dagger}$ For $\delta=1$, tree-level virtual graviton effects, and in particular the sign of their interference with the SM, are unambiguously determined. To compute it correctly it is convenient to focus on the small $t$ limit of Bhabha scattering, where the cross section does not depend on the spin of the colliding particles (eikonal approximation). As well known, in such a limit both electromagnetism and gravity give an attractive force between electrons and positrons, therefore their interference is constructive: graviton exchange increases the $\mathrm{SM} e^{+} e^{-} \rightarrow e^{+} e^{-}$cross section.

This argument allows to fix related processes, although it does not directly apply to $q \bar{q} \rightarrow q \bar{q}$ scattering: gluon exchange and graviton exchange have different colour indices, which suppress the would-be dominant interference at $|t| \ll s$. 
2. Electron fermion $\rightarrow$ electron fermion scattering can be obtained from 1 . by crossing $s \rightarrow t$, $t \rightarrow u$ and $u \rightarrow s$ in the squared amplitude:

$$
\begin{aligned}
\frac{d \sigma}{d t}\left(e f \rightarrow e^{\prime} f^{\prime}\right)= & \frac{s^{2}\left(\left|G^{L L}\right|^{2}+\left|G^{R R}\right|^{2}\right)+u^{2}\left(\left|G_{t}^{L R}\right|^{2}+\left|G_{t}^{R L}\right|^{2}\right)+t^{2}\left(\left|G_{u}^{L R}\right|^{2}+\left|G_{u}^{R L}\right|^{2}\right)}{16 \pi s^{2}} \\
& +\frac{1}{128 \pi s^{2}}\left[|\mathcal{S}(t)|^{2} G(t, u)+|\mathcal{S}(u)|^{2} G(u, t)\right. \\
& +2 \operatorname{Re} \mathcal{S}(t)^{*}\left(\left(G_{t}^{L R}+G_{t}^{R L}\right) u^{2}(3 t+4 u)+\left(G^{L L}+G^{R R}\right)(t+4 u) s^{2}\right) \\
& +2 \operatorname{Re} \mathcal{S}(u)^{*}\left(\left(G_{u}^{L R}+G_{u}^{R L}\right) t^{2}(4 t+3 u)+\left(G^{L L}+G^{R R}\right)(4 t+u) s^{2}\right) \delta_{e f} \\
& \left.+\frac{\operatorname{Re} \mathcal{S}(t) \mathcal{S}(u)^{*}}{2}(4 t+u)(4 u+t) s^{2} \delta_{e f}\right]
\end{aligned}
$$

Here

$$
G_{t}^{A B} \equiv \sum_{V=\gamma, Z, W} \frac{g_{A}(V \rightarrow \ell \bar{\ell}) g_{B}(V \rightarrow f \bar{f})}{t-M_{V}^{2}}, \quad G_{t}^{A B} \equiv \sum_{V=\gamma, Z, W} \frac{g_{A}(V \rightarrow f \bar{\ell}) g_{B}(V \rightarrow \ell \bar{f})}{u-M_{V}^{2}}
$$

and $G^{L L}=G_{t}^{L L}+G_{u}^{L L}, G^{R R}=G_{t}^{R R}+G_{u}^{R R}$. If $f=e d \sigma(e e \rightarrow e e)$ must be divided by 2 , if one wants to integrate it in the full range $-s<t<0$. At small $t \mathrm{SM}$ /graviton interference is destructive in $e e \rightarrow e e$ collisions and constructive in $e p \rightarrow e p$ collisions.

3. fermion antifermion $\rightarrow$ photon photon

$$
\frac{d \sigma}{d t}(f \bar{f} \rightarrow \gamma \gamma)=\frac{t^{2}+u^{2}}{64 \pi s^{2} t u N_{f}}\left|2 Q_{f}^{2}-t u \mathcal{S}(s)\right|^{2}
$$

where $N_{f}=1$ and $Q_{f}=-e$ if $f=e ; N_{f}=3$ if $f$ is a quark.

4. gluon gluon $\rightarrow$ photon photon

$$
\frac{d \sigma}{d t}(g g \rightarrow \gamma \gamma)=\frac{t^{4}+u^{4}}{512 \pi s^{2}}|\mathcal{S}(s)|^{2}
$$

5. quark antiquark $\rightarrow$ quark antiquark. Including only the gluon and the graviton contributions we find

$$
\begin{aligned}
\frac{d \sigma}{d t}(q \bar{q} \rightarrow q \bar{q})= & \frac{1}{16 \pi s^{2}}\left[\frac{8 g_{3}^{4}}{27 s^{2} t^{2}}\left(s^{2}-s t+t^{2}\right)\left(3 s^{2}+5 s t+3 t^{2}\right)\right. \\
& +\frac{2 g_{3}^{2}}{9} u^{2} \operatorname{Re}\left(\frac{4 t+s}{t} \mathcal{S}(s)^{*}+\frac{4 s+t}{s} \mathcal{S}(t)^{*}\right) \\
& \left.+\frac{\left|\mathcal{S}(s)^{2}\right| G(s, t)+\left|\mathcal{S}(t)^{2}\right| G(t, s)}{8}+\frac{\operatorname{Re} \mathcal{S}(s) \mathcal{S}(t)^{*}}{48}(4 s+t)(4 t+s) u^{2}\right]
\end{aligned}
$$

6. quark quark $\rightarrow$ quark quark is obtained by crossing the previous expression:

$$
\begin{aligned}
\frac{d \sigma}{d t}(q q \rightarrow q q)= & \frac{1}{32 \pi s^{2}}\left[\frac{8 g_{3}^{4}}{27 t^{2} u^{2}}\left(t^{2}-t u+u^{2}\right)\left(3 t^{2}+5 t u+3 u^{2}\right)\right. \\
& +\frac{2 g_{3}^{2}}{9} s^{2} \operatorname{Re}\left(\frac{4 u+t}{u} \mathcal{S}(t)^{*}+\frac{4 t+u}{t} \mathcal{S}(u)^{*}\right) \\
& \left.+\frac{|\mathcal{S}(t)|^{2} G(t, u)+|\mathcal{S}(u)|^{2} G(u, t)}{8}+\frac{\operatorname{Re} \mathcal{S}(t) \mathcal{S}(u)^{*}}{48}(4 t+u)(4 u+t) s^{2}\right]
\end{aligned}
$$

and $d \sigma(q q \rightarrow q q)=d \sigma(\bar{q} \bar{q} \rightarrow \bar{q} \bar{q})$. 
7. quark antiquark $\rightarrow$ quark $^{\prime}$ antiquark $^{\prime}$. The prime denotes that the two quarks are of different type. Including only the gluon and the graviton contributions we find

$$
\frac{d \sigma}{d t}\left(q \bar{q} \rightarrow q^{\prime} \bar{q}^{\prime}\right)=\frac{1}{16 \pi s^{2}}\left[\frac{4 g_{3}^{4}}{9 s^{2}}\left(s^{2}+2 s t+2 t^{2}\right)+\frac{|\mathcal{S}(s)|^{2}}{8} G(s, t)\right]
$$

8. quark quark ${ }^{\prime} \rightarrow$ quark quark $^{\prime}$ and quark antiquark ${ }^{\prime} \rightarrow$ quark antiquark $^{\prime}$. Including only the gluon and the graviton contributions we find

$$
\frac{d \sigma}{d t}\left(q \bar{q}^{\prime} \rightarrow q \bar{q}^{\prime}\right)=\frac{d \sigma}{d t}\left(q q^{\prime} \rightarrow q q^{\prime}\right)=\frac{1}{16 \pi s^{2}}\left[\frac{4 g_{3}^{4}}{9 t^{2}}\left(t^{2}+2 t u+2 u^{2}\right)+\frac{|\mathcal{S}(t)|^{2}}{8} G(t, u)\right]
$$

9. gluon gluon $\rightarrow$ gluon gluon:

$$
\begin{aligned}
\frac{d \sigma}{d t}(g g \rightarrow g g)= & \frac{1}{256 \pi s^{2}}\left[\frac{9 g_{3}^{4}\left(s^{2}+t^{2}+u^{2}\right)^{3}}{2 s^{2} t^{2} u^{2}}\right. \\
& -6 g_{3}^{2} \operatorname{Re}\left(\frac{t^{4}+u^{4}}{t u} \mathcal{S}(s)^{*}+\frac{s^{4}+u^{4}}{s u} \mathcal{S}(t)^{*}+\frac{s^{4}+t^{4}}{s t} \mathcal{S}(u)^{*}\right) \\
& +u^{4}\left(4|\mathcal{S}(s)|^{2}+\operatorname{Re} \mathcal{S}(s) \mathcal{S}(t)^{*}+4|\mathcal{S}(t)|^{2}\right) \\
& +t^{4}\left(4|\mathcal{S}(s)|^{2}+\operatorname{Re} \mathcal{S}(s) \mathcal{S}(u)^{*}+4|\mathcal{S}(u)|^{2}\right) \\
& \left.+s^{4}\left(4|\mathcal{S}(t)|^{2}+\operatorname{Re} \mathcal{S}(t) \mathcal{S}(u)^{*}+4|\mathcal{S}(u)|^{2}\right)\right]
\end{aligned}
$$

10. gluon gluon $\leftrightarrow$ quark antiquark

$$
\begin{aligned}
\frac{d \sigma}{d t}(g g \rightarrow q \bar{q})= & \frac{1}{16 \pi s^{2}}\left[\frac{-g_{3}^{4}}{24 s^{2} t(s+t)}\left(s^{2}+2 s t+2 t^{2}\right)\left(4 s^{2}+9 s t+9 t^{2}\right)\right. \\
& \left.-\frac{g_{3}^{2}}{8}\left(s^{2}+2 s t+2 t^{2}\right) \operatorname{Re} \mathcal{S}(s)^{*}-\frac{3}{16}|\mathcal{S}(s)|^{2} t(s+t)\left(s^{2}+2 s t+2 t^{2}\right)\right]
\end{aligned}
$$

The cross section for the inverse process is $d \sigma(q \bar{q} \rightarrow g g) / d t=(32 / 9) d \sigma(g g \rightarrow q \bar{q}) / d t$.

11. gluon quark $\rightarrow$ gluon quark can be obtained from the previous result by crossing $s \rightarrow t$, $t \rightarrow u$. Since we average over the colour of the initial state, we must also rescale $\sigma$ by a factor $8 / 3$. The result is:

$$
\begin{aligned}
\frac{d \sigma}{d t}(g q \rightarrow g q)= & \frac{1}{16 \pi s^{2}}\left[\frac{g_{3}^{4}}{9 s t^{2}(s+t)}\left(2 s^{2}+2 s t+t^{2}\right)\left(9 s^{2}+9 s t+4 t^{2}\right)+\right. \\
& \left.+\frac{g_{3}^{2}}{3}\left(2 s^{2}+2 s t+t^{2}\right) \operatorname{Re} \mathcal{S}(t)+\frac{1}{2}|\mathcal{S}(t)|^{2} s(s+t)\left(2 s^{2}+2 s t+t^{2}\right)\right]
\end{aligned}
$$

Also, $d \sigma(g q \rightarrow g q) / d t=d \sigma(g \bar{q} \rightarrow g \bar{q}) / d t$.

Finally we give the differential cross sections for the processes $e^{+} e^{-} \rightarrow \gamma G$ and $e^{+} e^{-} \rightarrow Z G$ :

$$
\frac{d \sigma}{d x d y}\left(e^{+} e^{-} \rightarrow V G\right)=\frac{S_{\delta-1} \alpha_{V}}{M_{D}^{2+\delta}} s^{\delta / 2} y^{\delta / 2-1} g_{V}(x, y, z)
$$


where $x=t / s, y=m^{2} / s, z=M_{V}^{2} / s, \alpha_{\gamma}=\alpha_{\mathrm{em}}, \alpha_{Z}=\alpha_{\mathrm{em}}\left(1-4 \sin ^{2} \theta_{\mathrm{W}}+\sin ^{4} \theta_{\mathrm{W}}\right) / 2 \sin ^{2} 2 \theta_{\mathrm{W}}=$ $0.36 \alpha_{\mathrm{em}}$, and

$$
\begin{aligned}
g_{Z}(x, y, z)= & \frac{1}{96 x^{2}(z-1-x+y)^{2}}\left\{-4 x^{6}(z-6)+12 x^{5}(z-6)(z-1+y)\right. \\
& -3 y^{2}(z-1)^{2} z(z-1+y)^{2}-2 x^{4}\left[-42+6 y^{2}(z-7)+78 z-58 z^{2}+6 z^{3}\right. \\
& \left.+y\left(75-114 z+17 z^{2}\right)\right]+3 x y(z-1)\left[1+y^{3}(z-1)-2 z+10 z^{2}-10 z^{3}+z^{4}\right. \\
& \left.+y^{2}\left(1-14 z+5 z^{2}\right)+y\left(13 z-1-25 z^{2}+5 z^{3}\right)\right]+4 x^{3}\left[12+y^{3}(z-12)-25 z\right. \\
& \left.+31 z^{2}-19 z^{3}+z^{4}+y^{2}\left(27-57 z+8 z^{2}\right)+y\left(-27+72 z-69 z^{2}+8 z^{3}\right)\right] \\
& +x^{2}\left[12 y^{4}+y^{3}\left(-33+90 z-13 z^{2}\right)+4(z-1)^{2}\left(3+5 z^{2}\right)\right. \\
& \left.\left.-4 y^{2}\left(-9+48 z-55 z^{2}+8 z^{3}\right)+y\left(-33+102 z-206 z^{2}+150 z^{3}-13 z^{4}\right)\right]\right\}, \\
g_{\gamma}(x, y)= & g_{Z}(x, y, 0)=\frac{[4 x(1+x-y)-y]\left[1+2 x(1+x)-2 x y+y^{2}\right]}{32 x(1+x-y)} .
\end{aligned}
$$

\section{References}

[1] N. Arkani-Hamed, S. Dimopoulos and G. R. Dvali, Phys. Lett. B 429 (1998) 263 hep-ph/9803315.

[2] S. Hannestad and G. G. Raffelt, Phys. Rev. D 67 (2003) 125008 [Erratum-ibid. D 69 (2004) 029901] hep-ph/0304029]; C. Hanhart, D. R. Phillips, S. Reddy and M. J. Savage, Nucl. Phys. B 595 (2001) 335 nucl-th/0007016.

[3] G. F. Giudice, R. Rattazzi and J. D. Wells, Nucl. Phys. B 544, 3 (1999) hep-ph/9811291.

[4] G. F. Giudice and A. Strumia, Nucl. Phys. B 663 (2003) 377 hep-ph/0301232.

[5] L. Randall and R. Sundrum, Phys. Rev. Lett. 83 (1999) 3370 hep-ph/9905221.

[6] N. Arkani-Hamed, S. Dimopoulos, G. R. Dvali and N. Kaloper, Phys. Rev. Lett. 84 (2000) 586 hepth/9907209.

[7] T. Gherghetta and M. E. Shaposhnikov, Phys. Rev. Lett. 85 (2000) 240 hep-th/0004014.

[8] N. Kaloper, J. March-Russell, G. D. Starkman and M. Trodden, Phys. Rev. Lett. 85 (2000) 928 hepph/0002001.

[9] K. R. Dienes, Phys. Rev. Lett. 88 (2002) 011601 hep$\mathrm{ph} / 0108115$.

[10] C. P. Bachas, JHEP 9811 (1998) 023 hepph/9807415; I. Antoniadis and C. Bachas, Phys. Lett. B 450 (1999) 83 hep-th/9812093.

[11] K.M. Cheung and W. Y. Keung, Phys. Rev. D 60 (1999) 112003 hep-ph/9903294.

[12] L3 collaboration, Phys. Lett. B 470 (1999) 281 hepex/9910056.

[13] ALEPH, DELPHI, L3, OPAL collaborations and LEP, SLD electroweak working groups, hep-ex/0312023.
The LEP2 data we fitted are summarized and combined in its chapter 8 . For the differential $e \bar{e} \rightarrow e \bar{e}$ cross sections we use instead the most recent results presented in: OPAL collaboration, CERN-EP/2003053 and in: ALEPH collaboration, "Fermion Pair production in e+e-collisions at 189-209 GeV and Constraints on Physics Beyond the Standard Model', paper in preparation.

[14] V. V. Anisimovsky et al. [E949 Collaboration], hepex/0403036.

[15] J. Bijnens and M. Maul, JHEP 0010, 003 (2000) hep$\mathrm{ph} / 0006042$.

[16] L. Vacavant and I. Hinchliffe, J. Phys. G 27, 1839 (2001).

[17] T. Han, D. L. Rainwater and D. Zeppenfeld, Phys. Lett. B 463, 93 (1999).

[18] W. T. Giele, E. W. N. Glover and D. A. Kosower, Nucl. Phys. B 403, 633 (1993).

[19] D. Stump, J. Huston, J. Pumplin, W. K. Tung, H. L. Lai, S. Kuhlmann and J. F. Owens, JHEP 0310, 046 (2003).

[20] K. M. Cheung and G. Landsberg, Phys. Rev. D 62, 076003 (2000).

[21] V. Kabachenko, A. Miagkov and A. Zenin, preprint ATL-PHYS-2001-012

[22] T. Binoth, J. P. Guillet, E. Pilon and M. Werlen, Eur. Phys. J. C 16, 311 (2000) and T. Binoth, private communication

[23] L. N. Chang, O. Lebedev, W. Loinaz and T. Takeuchi, Phys. Rev. Lett. 85, 3765 (2000).

[24] D. Atwood, S. Bar-Shalom and A. Soni, Phys. Rev. D 62 (2000) 056008 hep-ph/9911231. 


\section{Erratum}

The original version of the paper missed a simple but important difference between flat and slightly warped extra dimensions, related to the fact that warping reduces the number of Kaluza Klein gravitons making them more strongly coupled. In the phenomenologically interesting part of the parameter space the life-time $1 / \Gamma_{G} \sim \Lambda_{\pi}^{2} / m^{3}$ of gravitons $G$ with mass $m$ is so small that KK gravitons decay promptly. This is different from the case of flat extra dimensions, where $\Lambda_{\pi} \sim M_{\mathrm{Pl}}$ and gravitons decay far away from the detector, giving missing energy signals.

As a consequence:

1. There are no missing energy signals due to graviton emission in the model under consideration.

2. Graviton production ( $i \rightarrow G$ followed by $G \rightarrow f$ decays) contributes instead to $i \rightarrow f$ scatterings, where $i$ and $f$ denote initial and final SM particles.

The graviton amplitude $\mathcal{S}(s)$ was correctly computed here, but the new real contribution enhances its modulus squared, which becomes:

$$
\left\langle\left|\mathcal{S}^{2}\right|\right\rangle=(\operatorname{Re} \mathcal{S})^{2}+\frac{(\operatorname{Im} \mathcal{S})^{2}}{\epsilon}, \quad \epsilon=\left.\frac{\pi \Gamma_{G}}{2 \Delta m}\right|_{m=\sqrt{s}}=\frac{283}{960}\left(\frac{\sqrt{s}}{M_{5}}\right)^{3} .
$$

This enhancement of $|\mathcal{S}|^{2}$ is implicit in the equations of [1A, 2A] after averaging it over $\hat{s}$; the same averaging means that their result for $\mathcal{S}$ reduces to ours. The interpretation and consequences of this enhancement were described in $3 \mathrm{~A}]$.

In the original version of this work we argued that the interference between the SM and the graviton contribution $\mathcal{S}$ is significant only for elastic scatterings with $t$-channel virtual graviton exchange, such as $e^{-} e^{+} \rightarrow e^{-} e^{+}$. This remains true, but the enhanced real graviton amplitude now significantly contributes also to processes with $s$-channel graviton exchange, such as $e^{-} e^{+} \rightarrow \ell^{-} \ell^{+}$: both the elastic channel $(\ell=e)$ and the inelastic ones, $\ell=\{\mu, \tau\}$. This is illustrated in fig. 11, that replaces fig. 3 .

The bound from $e^{-} e^{+} \rightarrow e^{-} e^{+}$at LEP remains essentially unaltered: $M_{D}>2.5 \mathrm{TeV}$ at $99 \%$ confidence level (C.L). We find a new bound from the total LEP cross sections into $\mu^{-} \mu^{+}$and $\tau^{-} \tau^{+}$: $M_{D}>2.4 \mathrm{TeV}$ at $99 \%$ C.L. Combining the two bounds we find $M_{D}>3.3 \mathrm{TeV}$ at $95 \%$ C.L. The bound from missing energy signals, such as $e^{-} e^{+} \rightarrow \gamma G$ does not apply because such signals are not present.

At the LHC, the $p p \rightarrow j j$ signal (being dominated by $u u \rightarrow u u$ partonic scatterings) turns out to be negligibly affected by the enhancement in eq. (33) [3A], which should instead be significant for the $p p \rightarrow \ell^{-} \ell^{+}$and $p p \rightarrow \gamma \gamma$ signals.

\section{References}

[1A] A. V. Kisselev, Eur. Phys. J. C42 (2005) 217 hep-ph/0412376.

[2A] A. V. Kisselev, JHEP 0809, 039 (2008) arXiv:0804.3941.

[3A] R. Franceschini, G.F. Giudice, P.P. Giardino, P. Lodone, A. Strumia, arXiv:1101.4919 

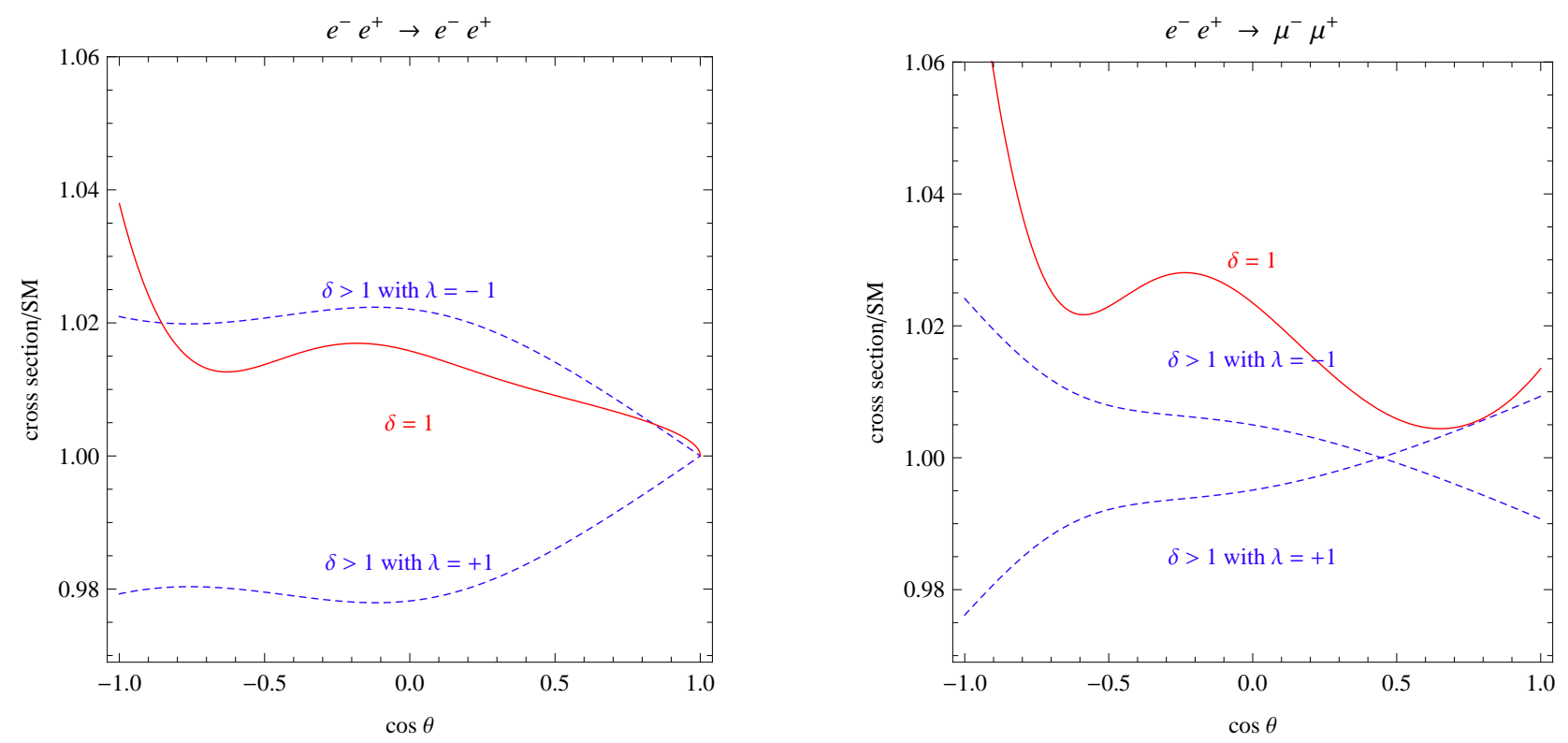

Figure 11: Corrections to $e^{+} e^{-} \rightarrow e^{+} e^{-}$(left) and $e^{+} e^{-} \rightarrow \mu^{+} \mu^{-}$(right) cross sections due to treelevel virtual and real exchange of gravitons with $\delta=1$ (red continuous line, for $M_{D}=2.5 \mathrm{TeV}$ ) and $\delta>1$ (blue dashed lines, for $\mathcal{S}=8 \lambda /(1.25 \mathrm{TeV})^{4}$ and $\left.\lambda= \pm 1\right)$. In the latter case the result depends on the UV cut-off, so that not even its sign can be reliably computed: we consider the two cases of constructive and destructive interference with the SM. For $\delta=1$ the effect is unambiguously fixed: it increases the Bhabha cross section, and gives a negligible correction to inelastic scatterings. 